\title{
Flow Field Analysis Around Pressure Shielding Structures
}

\author{
Máté Szőke, Nandita Hari† William J. Devenport; \\ Virginia Tech, Blacksburg, VA, 24061, USA \\ Stewart A. L. Glegg§ \\ Florida Atlantic University, Boca Raton, FL, 33431, USA \\ Tom-Robin Teschner ${ }^{\llbracket}$ \\ Cranfield University, Cranfield, MK43 OAL, UK
}

\begin{abstract}
The flow field around a series of streamwise rods, referred to as canopies, is investigated using two-dimensional two-component time-resolved particle image velocimetry (PIV) and large eddy simulations (LES) to characterize the changes in the flow field responsible for reducing the low and high-frequency surface pressure fluctuations previously observed. It was found that an axisymmetric turbulent boundary layer (ATBL) develops over the rods, whose thickness grows at a greater rate above the rods than below. This boundary layer reaches the wall below the rods at a point where previously a saturation was found in the low-frequency noise attenuation, revealing that the ATBL is responsible for the lowfrequency noise attenuation. The flow is displaced by the presence of the rods, particularly above them, which offset was primarily caused by the blockage of the ATBL. The flow below the rods exhibits the properties of a turbulent boundary layer as its profile still conforms to the logarithmic layer, but the friction velocity was found to drop. This viscous effect was found to be responsible for the high-frequency noise attenuation reported previously.
\end{abstract}

\section{Introduction}

The increasing demand for renewable energy has resulted in a dramatic growth of wind farms over the past few decades. The expansion of wind farms was followed by stringent noise emission regulations, which can limit future on-shore wind farm installations. Commercial aircraft and airports also face similar, stringent noise regulations which may restrict their operations or further growth. In all of these cases, the noise of concern originates from the turbulent boundary layer (TBL), more importantly, from the hydrodynamic pressure fluctuations (HPF) within the TBL. The HPF may excite the fuselage of the aircraft, generating airframe noise. As another example, trailing edge (TE) noise is generated as the HPF passes over the trailing edge and it scatters into far field noise $!^{1 / 3}$ Therefore, reducing the footprint of the HPF on aerodynamic surfaces, such as fuselage or wind turbine blades, is required to reduce noise emission levels of wind farms or commercial aircraft.

The problem of TE noise has already received extensive attention during the $1970 \mathrm{~s}$ and $1980 \mathrm{~s}, \frac{\sqrt[3]{6} 6}{6}$ but potential reduction methodologies have still been extensively studied over the past few decades. To date, some analytica ${ }^{\sqrt{3}-7}$ and empirical ${ }^{1}$ noise models are available to predict TE noise, but its reduction is still challenging. Potentially, computational fluid dynamics (CFD) and large eddy simulations (LES) may be used to predict far field noise in a number of studies 11 However, due to the rather high Reynolds numbers, in the order of millions, associated with wind turbine blades, the numerical estimation of far field TE noise is still computationally unfeasible. As a result, wind tunnel testing is often the only available resource to assess the noise emission levels of wind turbines and to develop TE noise reduction strategies.

\footnotetext{
*Research Associate, Kevin T. Crofton Department of Aerospace and Ocean Engineering, email: m.szoke@vt.edu

${ }^{\dagger} \mathrm{PhD}$ Candidate, Kevin T. Crofton Department of Aerospace and Ocean Engineering

$\ddagger$ Professor, Kevin T. Crofton Department of Aerospace and Ocean Engineering, AIAA Associate Fellow

§Professor, Department of Ocean and Mechanical Engineering, AIAA Associate Fellow

『 Lecturer, School of Aerospace, Transport and Manufacturing, Centre for Computational Engineering Sciences
} 
Several methodologies have been proposed to reduce TE noise over the past few decades. One of the

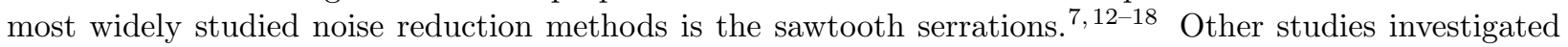

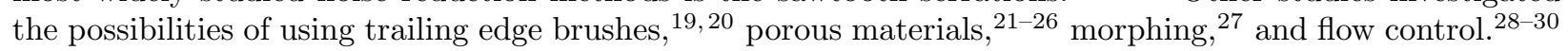
Recently, a new category of TE noise reduction strategies have been introduced, the trailing-edge finlets, where a series of vertical streamwise blades are used in the close vicinity of the TE to separate turbulence from the surface and to break up the turbulent structures before passing over the TE 3135 These mechanisms resulted in the reduction of surface pressure power spectral density downstream of the finlets, which, according to TE noise models, ${ }^{3}[\sqrt[6]{6}$ is a dominant parameter in the generation of TE noise.

Inspired by finlets, Gonzalez et al ${ }^{[36}$ proposed the use of straight, streamwise rods positioned above the surface. In this study, a series of solid rods were placed above a flat plate such that the rods were uniformly spaced and were parallel with the streamwise direction and were facing the flow. The resulting structures are called canopies and are inheriting the top edges of the fences used in finlets studies of Clark et al. and Afshariet al. 33 but canopies abandon the vertical support of finlets. The schematic of the problem is shown in Fig. 1. Previous studies ${ }^{36}{ }^{37}$ have shown that the canopies are efficient in reducing both the low- and high-frequency surface pressure fluctuations, therefore making them a promising tool for TE noise reduction.

Studies to date ${ }^{\sqrt[36]{38}}$ suggest that two main mechanisms are responsible for the reduction of surface pressure fluctuations when canopies are present over a solid wall. First, the low-frequency reduction is dominated by the shear sheltering effect triggered by the canopies, while high-frequency reductions are provided via a spectral shortcut mechanism that is responsible for enhancing the dissipation rate of smallscale turbulent structures. The low-frequency noise reduction was found to scale by $f h / U_{h}$, where $f$ is frequency, $h$ is the height of the canopies (distance from the wall to rod centerline), and $U_{h}$ is the mean velocity experienced by the leading edge of the rods, i.e. mean velocity at the height of the canopy in absence of the rods. In addition, the low-frequency attenuation of surface pressure was found to increase linearly from the leading edge of the canopies when scaled on canopy height, $h$. This increase, however, was found to saturate at $15 h-18 h$ downstream of the canopy leading edge, after which only a minor increase in noise attenuation was observed with further increasing streamwise location. The high-frequency noise reduction was driven by viscous effects, namely, scaling the high-frequency noise attenuation using $f \nu / U_{h}^{2}$, where $\nu$ is the viscosity of air, was shown to provide a reasonable, but not complete collapse $\frac{36}{37}$ of high-frequency noise reduction.
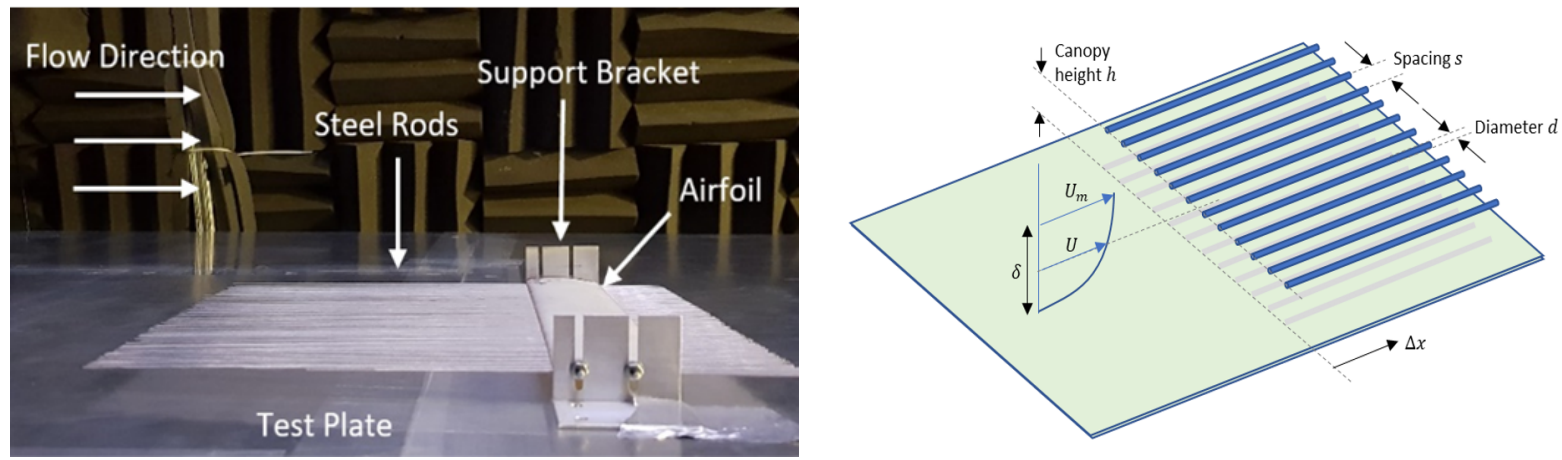

Figure 1. The canopies in the wall jet wind tunnel of Virginia Tech (left) and the schematic representation of the geometry (right). Figures adopted from Gonzalez et al. 36

While the noise reduction mechanisms of canopies stem from the fluid mechanical interaction between the rods and the turbulent boundary layer, the underlying flow properties have not yet been studied in detail. The aim of the present work, therefore, is to shed light on the flow field developing around the rods within the canopy structures. To assess and better understand these mechanisms in detail, high-speed time-resolved particle image velocimetry (PIV) experiments have been performed at Virginia Tech. The PIV investigations focused on measuring the flow-field between the rods, i.e. the field of view (FOV) was defined perpendicular to the wall. In parallel with the flow measurement efforts, numerical studies have been performed at Cranfield University, where large eddy simulations (LES) were carried out to capture the shear-sheltering and spectral shortcut mechanisms.

While the geometry of the underlying problem is relatively simple, it may be challenging to relate the 
developing flow field to a canonical flow. Still, some analogies may be anticipated which are now considered for discussion. First, the effect of shear sheltering has been widely studied analytically ${ }^{39} 41$ It was shown that, when considering a laminar boundary layer over a zero-pressure-gradient flat plate, the mean shear present in a Blasius boundary layer profile can prevent the penetration of gusts from the free-stream flow into the boundary layer. While the number of supporting experimental studies of shear sheltering is rather low, Hernon et al ${ }^{[42}$ have shown that the penetration depth (PD) of free-stream gusts, defined using the skewness of velocity fluctuations, scales favorably with the theoretical predictions. Still, experimental studies of shear sheltering have not yet been quantified experimentally in fully turbulent boundary layer flows. The phenomena may be interpreted by considering a turbulent eddy being convected within a TBL by the local mean velocity. Assuming this eddy is moving closer to the wall, i.e. to a fluid layer of lower mean velocity and higher velocity gradient, this eddy then may not be able to stay at this new wall distance because it is (a) still convecting faster than the local convection speed and (b) due to the tilting effect of the velocity gradient across the diameter of the eddy. With this assumption, we conclude that changing the velocity gradient and magnitude in a TBL will change the size of eddies that can be found at a certain wall distance.

The canopies are formed by a series of rods positioned streamwise with the mean flow. Hence another relevant characteristic flow field is the axisymmetric turbulent boundary layer developing over cylinders. Such a flow field is highly relevant for a wide range of applications, such as aircraft fuselage, submarines, rockets, and underwater sonar arrays, to mention a few, and therefore has been widely studied 43.48 It has been shown that an axisymmetric TBL becomes similar to a zero-pressure-gradient TBL when the ratio between the cylinder diameter $(a)$ and the thickness of the boundary layer $(\delta)$, i.e. $a / \delta$, increases. At low $a / \delta$ values, hairpin vortices generated near the wall in a TBL become comparable in size to the curvature of the surface $43 \sqrt[45]{45}$ This changes the gradient of the logarithmic layer when compared to a flat plate boundary layer. In addition, axisymmetric boundary layers were found to grow slower than flat plate boundary layers and they are also more stable than their flat plate counterpart. ${ }^{47}$ The boundary layer integral quantities, such as momentum thickness or displacement thickness, was found to be sensitive to misalignment between the freestream velocity and rod axis, but the boundary layer growth was shown to be insensitive to misalignment. Another important property of axisymmetric TBLs is that the skin friction is constant over the surface of the cylinder once the BL has reached a turbulent state. The constant skin friction can be confirmed using a momentum integral analysis $\underline{44} 46$

Overall, canopies may be considered a promising tool for reducing surface pressure fluctuations and therefore noise of wind turbines or commercial aircraft. Still, the developing flow field around the canopies is rather complex, partially because it is challenging to access experimentally and the flow around the rods may exhibit several flow features. In this paper, therefore, we make an attempt to characterize the flow field around the rods both experimentally and numerically to better understand the underlying noise reduction mechanism of canopies and to link changes in the flow field to noise reduction previously observed.

This paper is organized as follows. Section. II provides a detailed description of the canopy geometry and test facility followed by the details of the PIV setup. Section III provides a detailed description of the computational setup. Both experimental and numerical results are then presented in Section [V] with the findings of the paper summarized in Section $\mathrm{V}$.

\section{Experimental Approach}

This section provides the overview of the canopy geometries considered for analysis, followed by the description of the wind tunnel facility. A detailed description of the particle image velocimetry setup is then provided.

\section{A. The rig and measurement procedures}

While the present work builds on the studies of Gonzalez et al.,, 36 the geometry of the canopy has been changed. To improve optical access to the space between adjacent rods, the rod spacing $(s)$ has been increased to $s=6 \mathrm{~mm}$, while the open area ratio (OAR) of the rods was kept constant. Hence, the rod diameter $(d)$ was increased from $1 \mathrm{~mm}$ to $2 \mathrm{~mm}$. Two canopy heights $(h)$ have been considered for analysis, namely $h=6 \mathrm{~mm}$ and $h=15 \mathrm{~mm}$. The length of the canopies tested was $L=254 \mathrm{~mm}$, while their spanwise extent was $305 \mathrm{~mm}$. The aeroacoustic performance of these mid-size canopies has been tested prior to the PIV experiments. $\frac{37}{3 t}$ was found that these mid-size canopies $(d=2 \mathrm{~mm})$ show the same aeroacoustic 


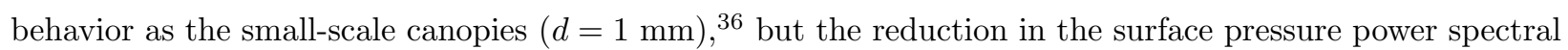
density was observed at lower frequencies and at slightly lower magnitudes. The overview of the test cases considered for analysis is given in Table 1 1 where baseline refers to a wall jet flow without the presence of the canopy structure.

Similarly to previous aeroacoustic investigations of canopies, $\frac{36 \mid 37}{37}$ experiments have been performed at the wall jet wind tunnel (WJT) of Virginia Tech. $\frac{49}{}$ In this tunnel, a $1 / 2$-inch tall nozzle blows air over a flat plate, hence its name wall jet, which results in a self-similar flow over the plate $\frac{50,52}{52}$ The boundary layer has two main flow regions, the near-wall flow exhibits the properties of a canonical zero pressure gradient turbulent boundary layer until a maximum velocity $\left(u_{m}\right)$ is reached at wall height $y_{m}$ in the velocity profile. Above this point, a two-dimensional shear layer flow dominates, which is characterized by energetic, largescale structures. Scaling the velocity profile using the peak velocity $u_{m}$, and the wall-normal distance $\left(y_{1 / 2}\right)$ where the mean velocity profile drops to half of $u_{m}$ provides a complete collapse of the velocity profile at all streamwise locations. Thanks to its self-similarity and simple geometry, some closed-form solutions are readily available to quantify the flow field of wall jet facilities. An example is given in the form of a calibration curve-fit for the WJT used in this study by Kleinfelter et al.$\underline{49}$ In addition, Gersten $\stackrel{\sqrt[50]{5}}{\text { presented }}$ a closed-form solution of wall jet velocity profile at different layers of the developing flow field over the wall. Experimental results obtained from the baseline (i.e. no canopy) case data are compared against the theoretical predictions of Gersten ${ }^{50}$ in Section IV. Finally, two jet speeds are considered for analysis, namely, the jet velocity, $u_{j e t}$, has been set to $30 \mathrm{~m} / \mathrm{s}$ and $50 \mathrm{~m} / \mathrm{s}$ during the experiments. The ambient conditions (pressure: $p_{a t m}$, temperature: $T$ ), and the dynamic pressure $(Q)$ at the nozzle exit were captured simultaneously with the PIV data acquisition. From these data, the jet speed could be kept constant within $\pm 0.1 \mathrm{~m} / \mathrm{s}$ during the tests, while the air density $\left(\varrho_{0}\right)$ and speed of sound $\left(c_{0}\right)$ could also be determined.

\begin{tabular}{|c|c|c|c|c|c|c|c|}
\hline Case ID & $\begin{array}{c}d \\
\mathrm{~mm} \\
\end{array}$ & $\begin{array}{c}s \\
\mathrm{~mm} \\
\end{array}$ & $\begin{array}{c}s / d \\
-\end{array}$ & $\begin{array}{c}h \\
\mathrm{~mm} \\
\end{array}$ & $\begin{array}{l}u_{j e t} \\
\mathrm{~m} / \mathrm{s}\end{array}$ & $\begin{array}{c}\text { FOV1 range } \\
\left(x_{\min } / h, x_{\max } / h,\right)\end{array}$ & $\begin{array}{c}\text { FOV2 range } \\
\left(x_{\min } / h, x_{\max } / h,\right)\end{array}$ \\
\hline Bas & - & - & - & - & 30,50 & $(-27 \mathrm{~mm}, 49 \mathrm{~mm})$ & $(47 \mathrm{~mm}, 123 \mathrm{~mm})$ \\
\hline D2S6H6 & 2 & 6 & 3 & 6 & 30,50 & $(-4.5,8.2)$ & $(7.8,20.5)$ \\
\hline D2S6H15 & 2 & 6 & 3 & 15 & 30,50 & $(-1.8,3.3)$ & $(3.1,8.2)$ \\
\hline
\end{tabular}

Table 1. List of test cases.

A high-speed PIV system is used to obtain instantaneous two-dimensional velocity data around the rods. The PIV system consists of a Phantom v2512 high-speed camera, a Photonics DM150-532 high-speed nanosecond laser, and a LaVision high-speed controller. Seeding was introduced to the flow at the suction side of the wind tunnel fan using an MDG MAX300APS type fog generator, providing mineral oil droplets with a diameter of 0.5-0.7 microns. A 300mm/F4 Nikon lens was used at an aperture of F5.6 to enable a detailed analysis of the flow field and provide a fine spatial resolution. The resulting FOV had a size of 80x50 $\mathrm{mm}$. The captured images of the flow were processed using the LaVision DaVis 10 software package and multi-pass vector calculation, namely, initially a larger, 64 x64 pix window was used with $50 \%$ overlapping to find velocity vectors, followed by a smaller, $16 \times 16$ pix window with $75 \%$ overlap. The smaller window size corresponds to a physical area of $1 \mathrm{~mm} \times 1 \mathrm{~mm}$. After overlapping the windows, the final spatial resolution of the data was $0.25 \mathrm{~mm}$. The temporal resolution of the system was limited by the camera, whose maximum sampling rate is $24 \mathrm{kFrames} /$ second. When operated in a double-frame mode, this enables us to capture 12,000 image pairs over a course of one second. Two types of data were captured. First, the sampling rate was set to 10,240 frame pairs per second, and two sets of 10,240 frame pairs were obtained, each corresponding to 1 second of flow time. Next, the sampling rate was set to 1024 frame pairs per second and 10,240 image pairs were captured, corresponding to 10 seconds of data. The former dataset enables a fine temporal flow analysis, while the latter enables spatial analysis of the flow. From a statistical convergence analysis of Reynolds stresses within the FOV, it was found that 2 seconds of flow data provides a $98 \%$ convergence of mean Reynolds stresses.

Optical imaging of the flow was performed by positioning the laser sheet between the rods, at the midspan of the canopies, while the camera was located outside of the wall jet flow to the side of the canopies, see Fig. 2. The 300mm lens and the narrow FOV enabled us to position the camera's line of sight such that it lined up with the spanwise direction of the rods. This enabled simultaneous data acquisition both below and above the rods. The thickness of the laser sheet within the FOV was $1.5 \mathrm{~mm}$. Due to the small extent 
of the FOV, two FOVs were used, whose locations in both dimensional and non-dimensional forms are given in Table 1. The coordinate system used in this work is also given in Fig. 2 with $x=0 \mathrm{~mm}$ corresponding to the leading edge of the canopies and $y=0 \mathrm{~mm}$ denotes the location of the wall.

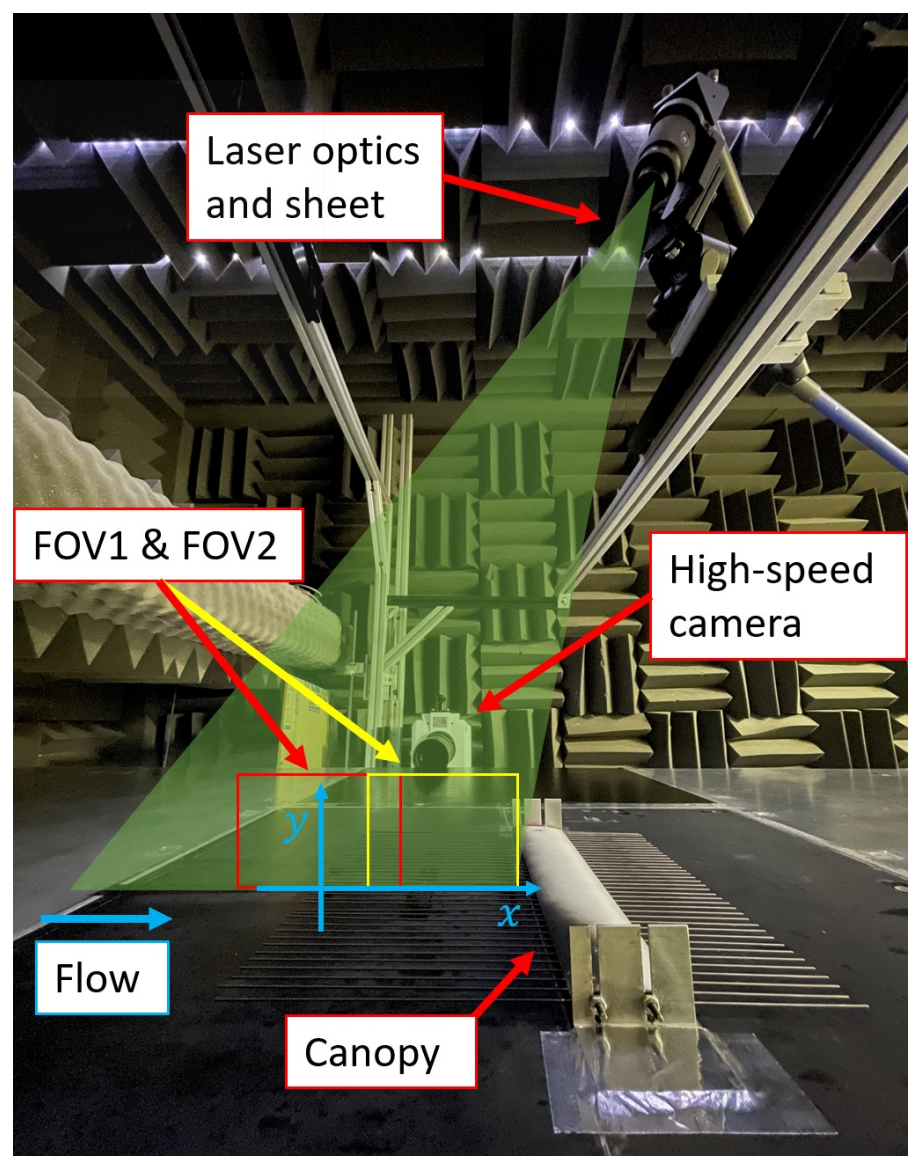

Figure 2. The canopy and the PIV system and in the wall jet wind tunnel.

\section{Computational Approach}

In the following section, we provide an overview of the numerical setup that has been employed for the LES studies. The usage of LES at high Reynolds numbers necessitates the usage of a reduced domain which we describe here in detail, followed by a description of the governing equations and boundary conditions.

\section{A. Computational domain and mesh}

In our LES studies, we focus on the D2S6H6 case setup in Table 1 for $u_{j e t}=30 \mathrm{~m} / \mathrm{s}$. We determined that a LES study, simulating the full wind tunnel test section at this Reynolds number would be prohibitively expensive due to the restrictive $x^{+}, y^{+}$and $z^{+}$requirements for wall-resolved simulations. Estimations of the computational time, taking available computing resources into account, were of the order of several months. To circumvent these restrictions, we could employ a hybrid RANS-LES turbulence model such as detached-eddy simulations (DES), where the subgrid-scale (SGS) modeling of a pure LES study is replaced by a RANS model, effectively relaxing the meshing requirements close to the wall. While this would speed up the computation, we would loose near-wall information which is now covered in an isotropic RANS layer, which potentially may further contaminate turbulent eddies close to the viscous sublayer within the TBL. Furthermore, hybrid RANS-LES simulations are not without their difficulties and the initial development of DES has shown to be prone to grid-induced separations which have then lead to the development of delayed detached-eddy simulations (DDES), improved delayed detached-eddy simulations (IDDES), and 


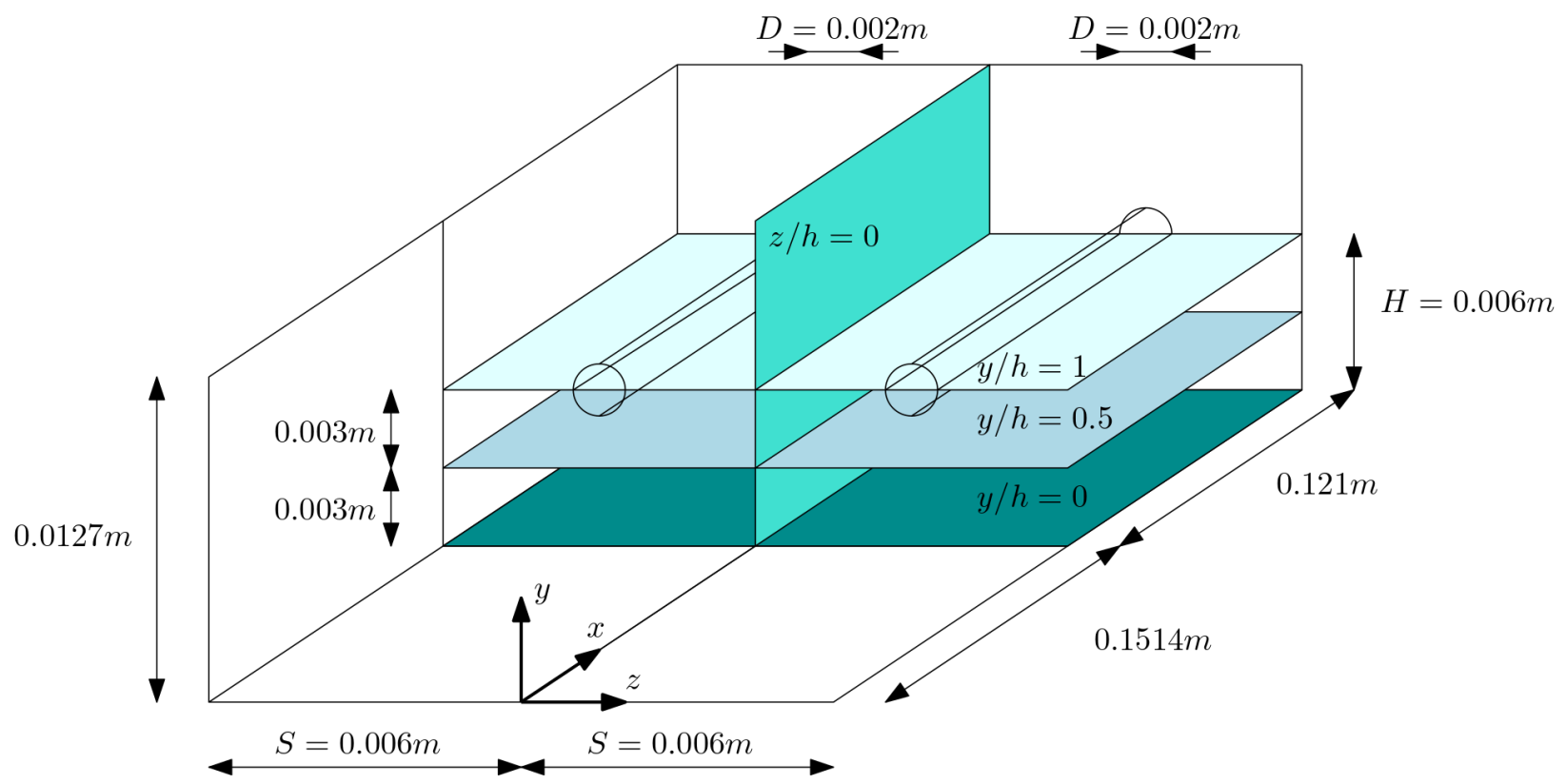

Figure 3. Computational domain used for the large eddy simulation. The planes at $y / h=0, y / h=0.5, y / h=1$ and $z / h=0$ are shown at which velocity and pressure information are collected.

scale-adaptive simulations (SAS). Thus, in order to reduce the modeling uncertainties, we decided to focus our computational approach on a reduced geometry, where two rods can be found in the domain both embedded within a TBL while the large scales were resolved through pure LES. The setup for this reduced domain can be seen in Fig. 3. Here, we also show the planes $y / h=0, y / h=0.5, y / h=1$ and $z / h=0$ at which we collect the velocity components and pressure for comparisons with the experimental PIV studies.

The computational mesh was constructed using a block-structured H-grid topology with the rods wrapped around an O-grid. Throughout the domain, we ensured that our spacing along the wall was set to $30<x^{+}<$ $50, y^{+} 1$, and $z^{+} 20$ which follows recommended grid generation practices for wall-resolved LES studies. ${ }^{[53}$ Overall, the mesh contains 4.16 millions cells and a close-up view near the beginning of the rods is shown in Fig. 4 .

\section{B. Governing equations}

The governing equations for a $3 \mathrm{D}$, incompressible flow are given by

$$
\begin{array}{r}
\frac{\partial \bar{u}_{i}}{\partial x_{i}}=0, \\
\frac{\partial \bar{u}_{i}}{\partial t}+\frac{\partial \bar{u}_{i} \bar{u}_{j}}{\partial x_{j}}=-\frac{1}{\rho} \frac{\partial \bar{p}}{\partial x_{i}}+2 \nu \frac{\partial \bar{S}_{i j}}{\partial x_{j}}-\frac{\partial \tau_{i j}}{\partial x_{j}},
\end{array}
$$

where we have defined the rate-of-strain tensor as

$$
\bar{S}_{i j}=\frac{1}{2}\left(\frac{\partial \bar{u}_{i}}{\partial x_{j}}+\frac{\partial \bar{u}_{j}}{\partial x_{i}}\right) .
$$

In Eq. 22,$\tau_{i j}$ represents the stresses that result from the filtering operation of the Navier-Stokes equations. These in turn are given by

$$
\tau_{i j}-\frac{1}{3} \tau_{k k} \delta_{i j}=-2 \nu_{S G S} \bar{S}_{i j}
$$

where $\tau_{k k}$ can be omitted. Thus, solving the above set of equations requires knowledge of the SGS viscosity. Classical approaches to calculating $\nu_{S G S}$ are based on algebraic closures such as the Smagorinsky 


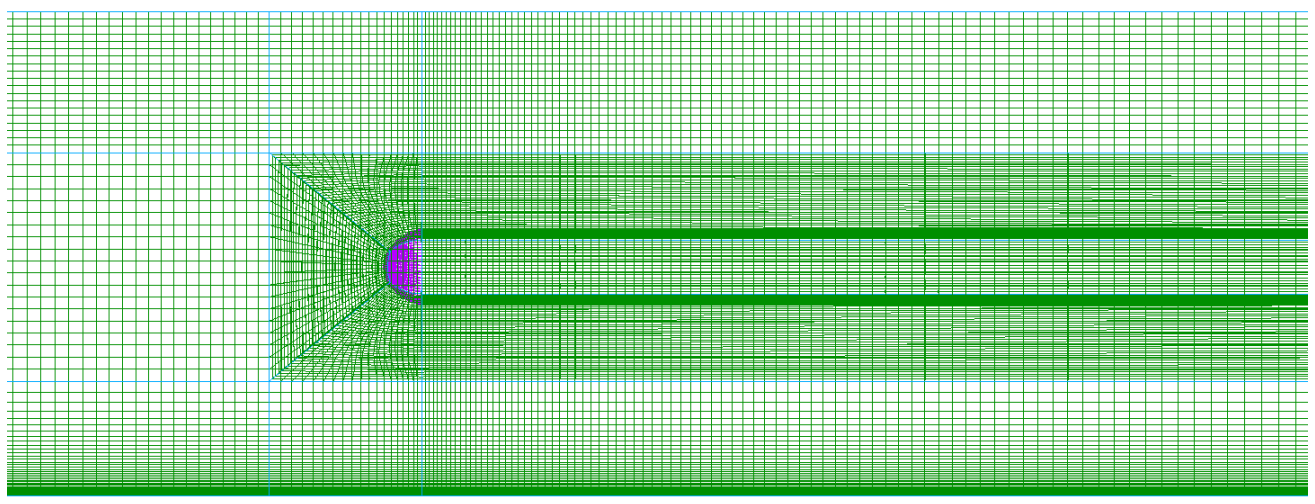

Figure 4. Computational mesh used for the LES studies

model,$\sqrt[54]{5}$ relying on experimental closure coefficients that may be dynamically tuned to improve the applicability of this SGS model. For wall-bounded flows, however, the wall-resolved local eddy-viscosity (WALE) mode ${ }^{\sqrt{55}}$ has shown to yield the correct wall asymptotic behavior by using local velocity gradient information. It is given by

$$
\nu_{S G S}=L_{S}^{2} \frac{\left(S_{i j}^{d} S_{i j}^{d}\right)^{3 / 2}}{\left(\bar{S}_{i j} \bar{S}_{i j}\right)^{5 / 2}+\left(S_{i j}^{d} S_{i j}^{d}\right)^{5 / 4}},
$$

where $L_{S}$ is defined as

$$
L_{S}=\min \left(\kappa d, C_{w} V^{1 / 3}\right) .
$$

Here we have the von Kármán constant $\kappa=0.41, d$ being the distance from the wall, $V$ is the volume of a given computational cell and $C_{W}=0.325$ which is referred to as the WALE constant. We further have to introduce

$$
S_{i j}^{d}=\frac{1}{2}\left(\bar{g}_{i j}^{2}+\bar{g}_{i j}^{2}\right)-\frac{1}{3} \delta_{i j} \bar{g}_{k k}^{2},
$$

where the additional velocity gradient tensor is defined as

$$
\bar{g}_{i j}=\frac{\partial \bar{u}_{i}}{\partial x_{j}} .
$$

\section{Boundary conditions}

As the computational domain, shown in Fig. 3, is situated within the TBL and the simulations resolve only a small portion of interest of the wind tunnel experiments, imposing boundary conditions that are suitable for LES studies becomes challenging. While experimental data is available $0.1514 m$ upstream of the rods, i.e. where the inlet section of the computational domain is situated, simply imposing the measured mean velocity profile would ignore any turbulent flow structures present within the TBL. In order to match the experimental data at the inlet, we make use of a random flow generator proposed by Smirnov et al., 56 which provides anisotropic turbulent and divergence-free fluctuations. In order to match the experiments, these random flow structures take into account the profiles for the turbulent intensity and the turbulent length scales at the inlet. This approach provides a sufficient level of turbulence at the inlet, matching that of the TBL measured in the experiment and we provide $0.1514 \mathrm{~m}$ of development length in order for the synthetically generated turbulence to become physical.

The sides of the domain are treated as periodic boundaries and we impose outflow condition at the end of the domain. In order to channel the TBL from the inlet towards the rods without imposing gradients at 
the top wall, we set this section to a symmetry condition, i.e. $\partial \bar{u}_{x} / \partial y=0$. We then change to an outlet boundary condition above the rods to allow fluid to exit the domain, as the experimental studies have shown that the flow experiences an upward motion as a result of the rods being present.

\section{Solver and data collection setup}

We use a bounded second-order implicit time integration for the time derivative and bounded central differencing for the advective term in Eq. 2. All other quantities are approximated using second-order approximations. The timestep is adaptively calculated based on a CFL number of 10, which corresponds to a $\Delta t \approx 10^{-5}$ second. The pressure velocity coupling for this incompressible simulation is achieved through the SIMPLE algorithm. Eqs. (1)-(2) are solved using a multigrid approach with a Gauss-Seidel smoother.

The simulation is run until statistical convergence is reached and we use the integrated velocity components and pressure on the $y / h=0, y / h=0.5, y / h=1$ and $z / h=0$ planes as the indicator to judge such convergence. After approximately 10 flow through times, we achieve statistical convergence and then run the simulation for an additional 2 seconds of simulated time, which corresponds to approximately 40 flow through times. The 2 seconds flow time was chosen based on the PIV analysis, where it was previously found to provide a $98 \%$ convergence of mean Reynolds stresses. Our sampling frequency is $10 \mathrm{kHz}$ to approximately match the PIV data. A total of $2.4 \mathrm{~TB}$ of raw data is gathered during ten days of calculations on 64 cores on Cranfield University's HPC cluster Delta.

\section{Results}

This section provides the results obtained from PIV experiments and LES simulations. We first review the PIV results of the baseline case followed by the analysis of the flow around the canopies. Data is then shown for the LES case with the presence of the rods.

\section{A. Baseline case}

This section provides a detailed description of the baseline, wall jet flow in the streamwise region where the canopies are installed. We first present the mean velocity, Reynolds shear stresses, and turbulent kinetic energy (TKE) at FOV1 and FOV2. This is followed by the description of how the friction velocity was found using the velocity profiles. The baseline dataset is then validated against the theoretical model of Gersten! 50

Figure 5 shows the contour maps of the mean streamwise velocity $(\bar{u})$, along with the Reynolds stress $\left(R_{i j}\right)$ contour maps and the TKE calculated using the first two principal Reynolds stresses for FOV1 and FOV2 obtained at $u_{j e t}=50 \mathrm{~m} / \mathrm{s}$. The dot markers in Figure 5(a) indicate the streamwise locations where the same type of data $\left(\bar{u}, R_{i j}\right)$ are shown in Fig. 6. The data presented in Figs. 5 and 6 were calculated using the low sampling rate PIV dataset (1024 frame pairs per second). As can be expected from the wall jet flow, the mean velocity increases from the wall $(y / h=0)$, i.e. shows a boundary layer-like behavior, until a maximum velocity value $\left(u_{m}\right)$ is reached. Above this point, the velocity begins to decrease with increasing $y$. This area was reported to observe a shear layer-like behavior $49[50[52$ The wall-normal velocity is shown in Fig. 6(a) and it is found that a low magnitude of vertical velocity is present within the flow field, but this velocity magnitude is comparable to the uncertainty of the PIV dataset, which was found to be below $0.7 \mathrm{~m} / \mathrm{s}$ for instantaneous velocity data and $0.4 \mathrm{~m} / \mathrm{s}$ for the time-averaged data (i.e. $\bar{u}$ or $\bar{v}$ ).

Both $R_{u u}$ and $R_{v v}$ Reynolds stresses are positive across the entire domain and would be expected to show an increasing behavior until $y_{1 / 2} \sqrt{49 \sqrt[50]{52}}$ is reached. The $R_{u v}$ shear stress, on the other hand, is negative below $y / h \approx 1.5$ and positive above. This is due to the shear layer-like behavior of the flow, where the center of the turbulent gusts are located, on average, at $y / h \approx 1.5$. Within a conventional zero-pressure gradient turbulent boundary layer, $R_{u v}$ Reynolds stress would not be expected to change sign.

From the mean velocity data, it was possible to obtain the friction velocity $u_{\tau}$ by minimizing the discrepancy between Spalding's equation and the experimental data over a range of dimensionless wall distances $\left(y^{+}\right)$. Spalding's equation, given in Eq. 9 is a function describing the viscous layer, buffer layer, and loglayer of a zero-pressure gradient turbulent boundary layer flow. The dimensionless velocity $\left(u^{+}=u / u_{\tau}\right)$ and dimensionless wall distance $\left(y^{+}=y u_{\tau} / \nu\right)$ was defined in a conventional way. The discrepancy was minimized between the experimental data and Eq. 9 within $30<y^{+}<200$ by varying $u_{\tau}$ and applying a small offset to $y$, which was limited to the half of the interrogation window size $\left(y_{\text {offset }}<0.125 \mathrm{~mm}\right)$. The vertical offset of the data $\left(y_{\text {offset }}\right)$ was defined as a linear function of the streamwise location, $x$, to account 


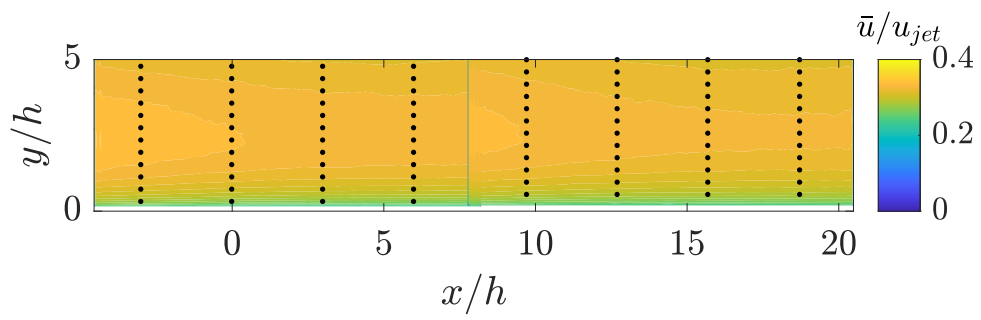

(a) Contour map of velocity magnitude.
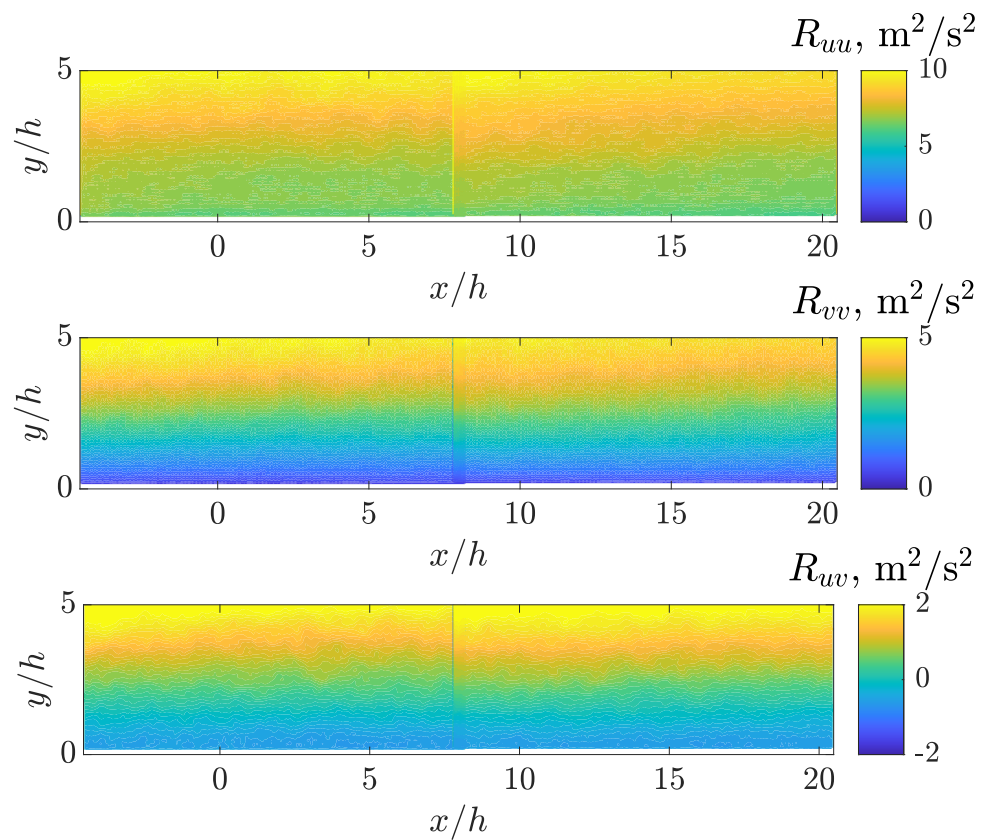

(b) Contour maps of Reynolds stresses.

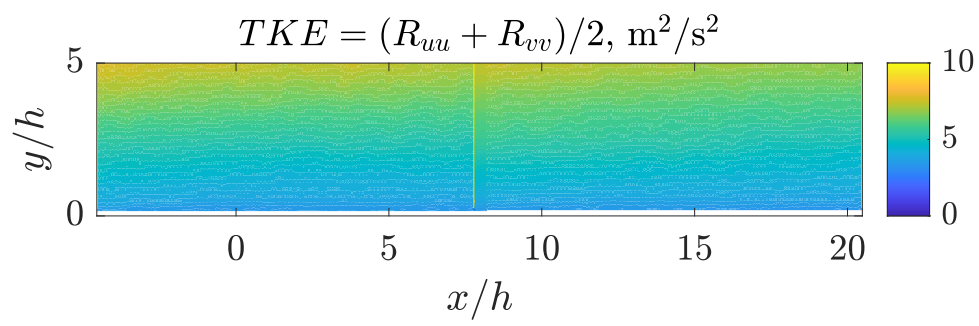

(c) Contour map of turbulent kinetic energy, TKE.

Figure 5. Mean $x$ velocity $(\bar{u})$, Reynolds stress $\left(R_{i j}\right)$ and turbulent kinetic energy (TKE) contour maps of the baseline case. Spatial coordinates were normalized using $h=6 \mathrm{~mm}$ for comparison purposes with the canopy data. The dot markers in the $\bar{u} / u_{\text {jet }}$ contour map indicate locations where one-dimensional plots are also considered, namely at $x / h=-3,0,3,6,9.7,12.7,15.7,18.7$. 
for any minute tilting of the camera. The misalignment between the camera and the wall was assessed prior to data acquisition using the reflection of the laser sheet from the wall, which was a straight line within 1-2 pix in the raw images. From this, the friction velocity $\left(u_{\tau}\right)$ was calculated at all streamwise locations $(x)$ within FOV1 and FOV2.

Dimensionless velocity results for the baseline case at $u_{\text {jet }}=50 \mathrm{~m} / \mathrm{s}$ for FOV1 and FOV2 are shown in Fig. 7 at the same streamwise locations where data is presented in Fig. 6. In general, a good agreement was found between the logarithmic layer and experimental data. The lowest $y^{+}$value where data was available from the PIV images was approximately $y^{+}=50$. In agreement with previous observations, 50,52 the dimensionless velocity profile begins to decrease and departs from the law of the wall above the logarithmic layer.

$$
y_{S}^{+}=u^{+}+\mathrm{e}^{-\kappa B}\left[\mathrm{e}^{\kappa u^{+}}-1-\kappa u^{+}-\frac{1}{2}\left(\kappa u^{+}\right)^{2}-\frac{1}{6}\left(\kappa u^{+}\right)^{3}\right],
$$

Finally, the results from the baseline case are compared against the theoretical model of Gersten ${ }^{50}$ in Fig. 8, where the skin friction coefficient $\left(c_{f}\right)$, the ratio of peak velocity location $\left(y_{m}\right)$ to the overall boundary layer thickness $\left(y_{1 / 2}\right)$, and the overlap and outer layer profiles are shown. For a detailed description of the theoretical model, we refer to Gersten, $\frac{50}{6}$ while the expressions used to generate Fig. 8 are given in Eqs. (10)17). Data are shown for 3 jet speeds in Fig. 8 , while the overall boundary layer thickness $y_{1 / 2}$ was found from the power-law curve fits of Kleinfelter et al.,$\frac{49}{}$ which relies on the jet velocity and streamwise distance from the jet nozzle exit plane. The leading edge of the canopies, corresponding to $x=0 \mathrm{~mm}$, was located at $1.28 \mathrm{~m}$ downstream of the nozzle.

$$
\begin{array}{r}
c_{f}=\frac{2 \tau_{w}}{\varrho_{0} u_{m}^{2}} \\
R e_{m}=\frac{u_{m} y_{m}}{\nu} \\
\bar{u}\left(y_{1 / 2}\right)=u_{m} / 2 \\
\eta=y / y_{m} \\
f^{\prime}=\frac{u_{m}-u}{u_{\tau}} \\
\bar{\eta}=\frac{y-y_{m}}{\Delta} \\
\Delta=\frac{y_{1 / 2}-y_{m}}{0.8814} \\
\dot{F}=\frac{u}{u_{m}}
\end{array}
$$

Overall, a very good agreement is found between the flow model of Gersten $\sqrt{50}$ and the PIV dataset. The results show that the skin friction coefficient is slightly underestimated compared to the model, but according to Gersten ${ }^{50} c_{f}$ is expected to decrease as $R e_{m} \rightarrow \infty$. Considering that the currently used WJT is a rather large research-oriented wall jet facility, the discrepancy between the theoretical and experimental data is acceptable. The $y_{m} / y_{1 / 2}$ curves show a perfect agreement with the model. Finally, the overlap layer, spanning between the viscous sublayer and $y_{m}$ and the outer layer, defined above $y_{m}$, are shown. Again, a good agreement is found between the theory and experiments, especially in the overlap layer. The wall-normal extent of the FOV allowed only a small portion of the outer layer to be captured, but a good agreement has been found between the experiments and the theory. The data shows a somewhat higher spread with increasing wall-distance $(\bar{\eta})$, but this may be accounted for the fact that the flow in the outer layer is highly turbulent, and long acquisition times (16 s) may be required to capture a better statistical convergence within this portion of the flow.

\section{B. Canopy flow field}

This section provides a detailed description of the flow around the canopies. Two cases are considered, D2S6H6 and D2S6H15 with both datasets captured at $u_{j e t}=50 \mathrm{~m} / \mathrm{s}$. Data is first presented concerning the 

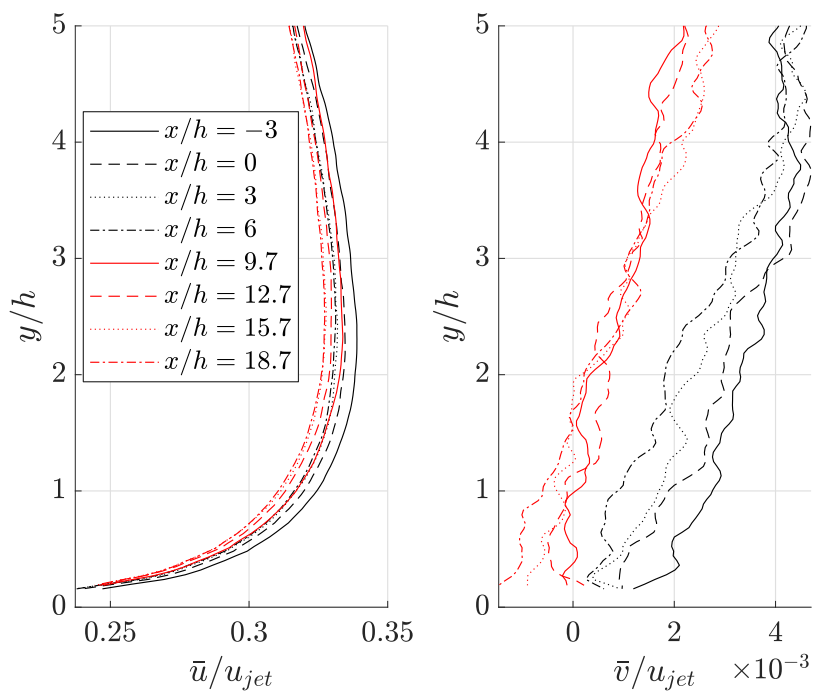

(a) Boundary layer velocity profiles.
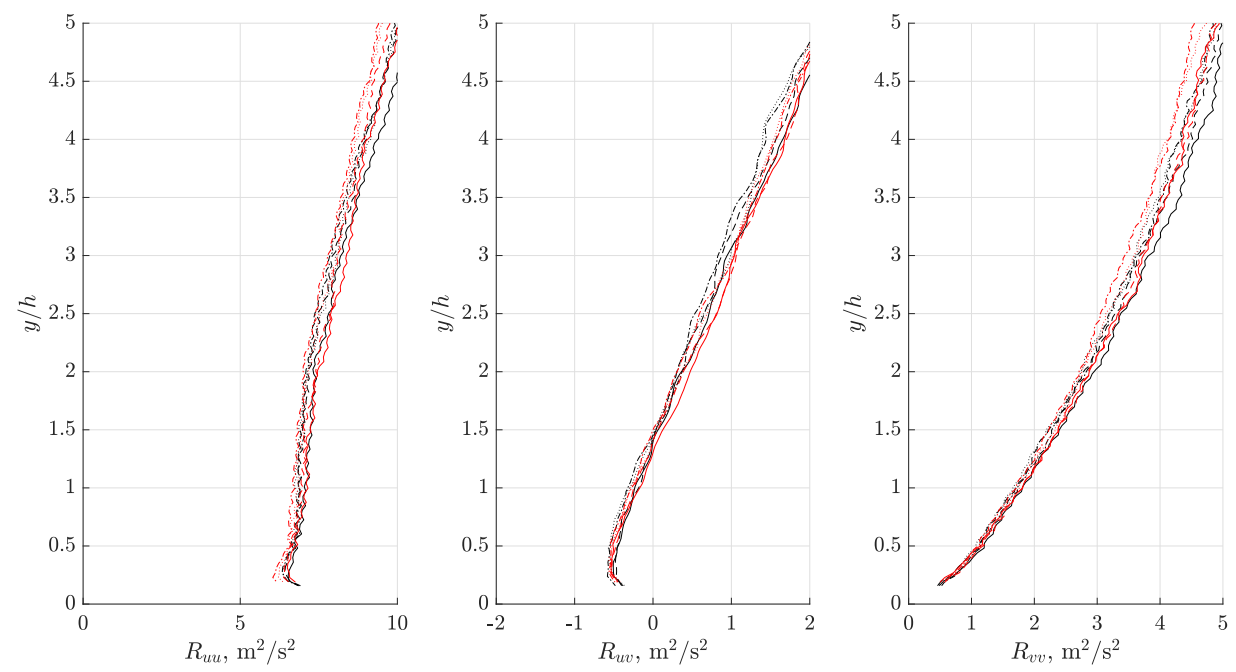

(b) Reynold shear stress profiles.

Figure 6. Mean $x$ and $y$ velocity $(\bar{u}, \bar{v})$ and Reynolds stress $\left(R_{i j}\right)$ profiles of the baseline case at $u_{j e t}=\mathbf{5 0} \mathbf{m} / \mathbf{s}$. Spatial coordinates were normalized using $h=6 \mathrm{~mm}$. The streamwise locations shown in the figure are marked in Fig. 5 (a). 


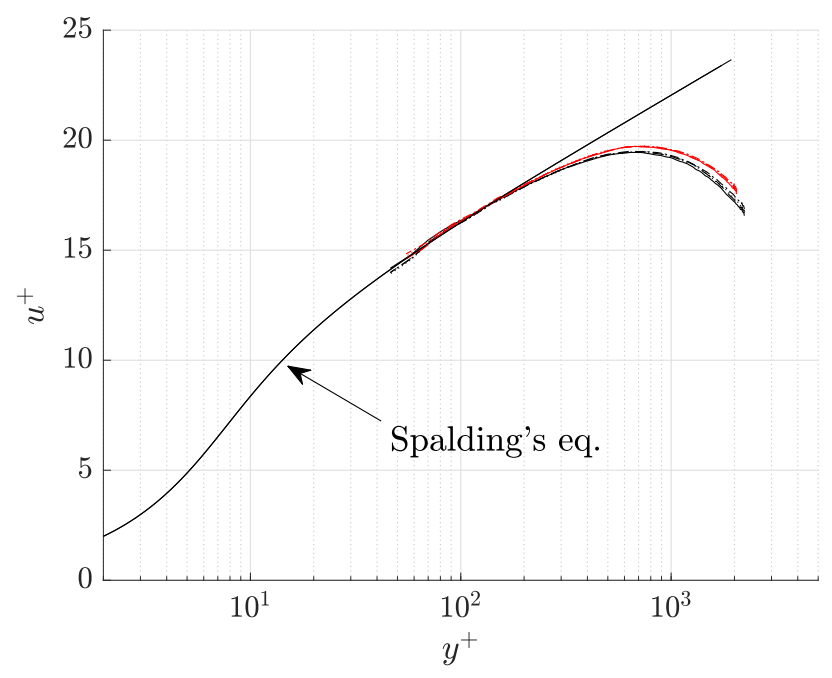

Figure 7. Spalding's dimensionless velocity profile and dimensionless velocity profiles for the baseline case obtained from FOV1 (black) and FOV2 (red). For legend, see Fig. 6(a).

mean velocity field around the canopies to assess their effect on the flow, followed by analyzing the changes in the Reynolds stresses and friction velocity.

The mean velocity contours and streamlines within FOV1 and FOV2 are shown in Fig. 9 for case D2S6H6. A few interesting observations can be made. The flow in the vicinity of the canopy leading edge (entry condition), at $x / h=0$, does not exhibit any separation or sudden changes in the contour levels, furthermore, the small disruption of the flow around the canopy leading edge is rather localized. The streamlines above the rods $(x / h>1)$ reveal that the flow is slightly drifting away from the wall and the inclination of the streamlines is approximately constant across both FOVs. This suggests that the introduction of the canopies has a flow displacement effect. Another important observation is that the streamlines below the rods $(x / h<1)$ are rather parallel, suggesting that the upwash (or blockage effect) above the rods is not necessarily originated from below the rods.

To better visualize the effect of the canopies on the mean flow field, the velocity at various streamwise locations $(x / h)$ are shown in Fig. 10(a) both as line-plots and contour plot. In the left sub-figure, we observe the velocity profiles without (baseline) and with canopy D2S6H6, while on the right sub-figure, the velocity deficit $\left(\Delta \bar{u}=u_{\text {baseline }}-u_{\text {canopy }}\right)$ is shown as a contour plot within FOV2. The canopies decelerate the flow around the rods and a velocity defect is observed in the velocity profiles. This deficit increases linearly in wallnormal extent both below and above the rods, but a steeper growth is found above the rods. This velocity deficit is in agreement with the deficit scaling of axisymmetric turbulent boundary layers, ${ }^{43}[4]$ with the main exception that in previous studies the rods were immersed into a uniform oncoming flow ( $\bar{u}=$ constant), while in the current case the rods interact with a turbulent boundary layer flow. After determining the boundary layer thickness around the rods as $\Delta \bar{u}(\delta)=0.01 \max (\Delta \bar{u})$, and using $(y-h) / \delta$ as a spatial scale, results of $\delta$ can be seen in Fig. 10(b) above the rods, where $\delta_{\text {top }}$ refers to the boundary layer thickness above the canopy $(y / h>1)$. A left sub-figure presents all $x$ locations as a one-dimensional plot, while the right-sub figure shows the same data but as a function of $x / h$. A good collapse of the data is now observed using the boundary layer deficit scaling, which confirms the presence of an axisymmetric turbulent boundary layer around the rods.

The boundary layer above $\left(\delta_{t o p}\right)$ and below $\left(\delta_{b o t}\right)$ the rods (D2S6H6) are shown as a function of streamwise distance $(x / h)$ in Fig. 11(a), while theoretical predictions of $\delta_{\text {theory }}$ are also presented using the empirical curve-fits of Jordan $\frac{46}{1}$ In agreement with Fig. 10 , the boundary layer both below and above the rods show a linear increase, with a steeper gradient above the rods. In addition, $\delta$ values below and above the rods provide a mean value resulting approximately the predictions of Jordan, $\frac{46}{,}$ i.e. $\left(\delta_{b o t}+\delta_{\text {tot }}\right) / 2=\delta_{\text {theory }}$. Again, this observation confirms the presence of an axisymmetric turbulent boundary layer around the rods. Finally, another observation is that $\delta_{b o t}$ saturates at $x / h \approx 15$. In the previous studies of canopy flows, the surface pressure attenuation caused by canopies at low frequencies was found to increase linearly (similarly to $\delta$ ) until $x / h \approx 16$, beyond which a plateau of peak attenuation was observed ${ }^{[36}$ Therefore, data suggest that 


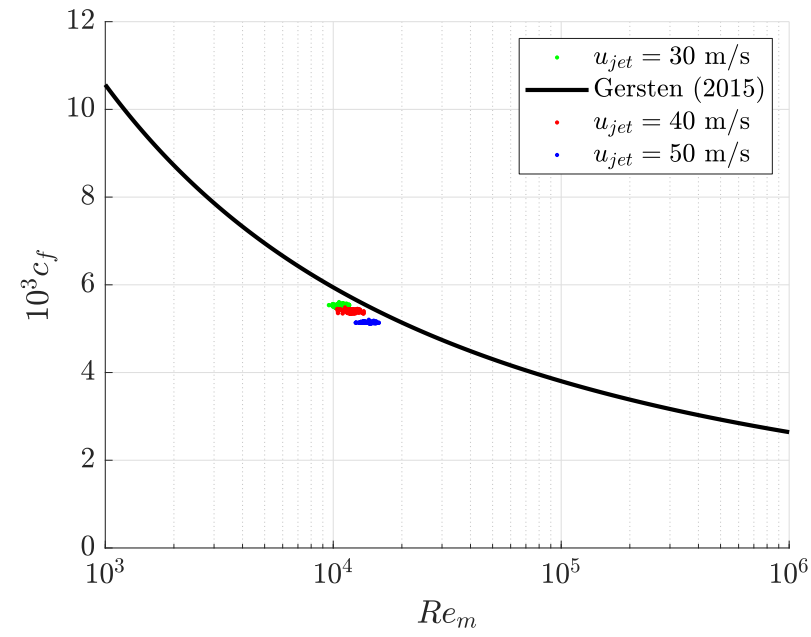

(a) Skin friction coefficient.

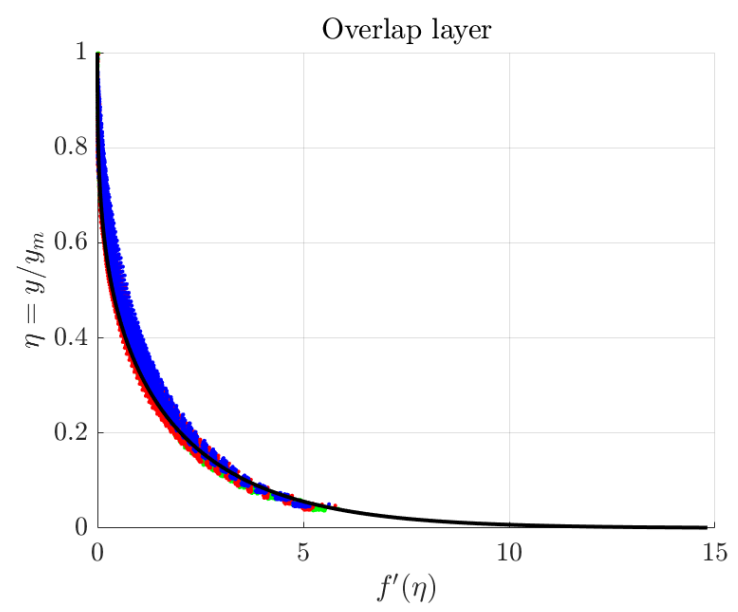

(c) Boundary layer overlap layer.

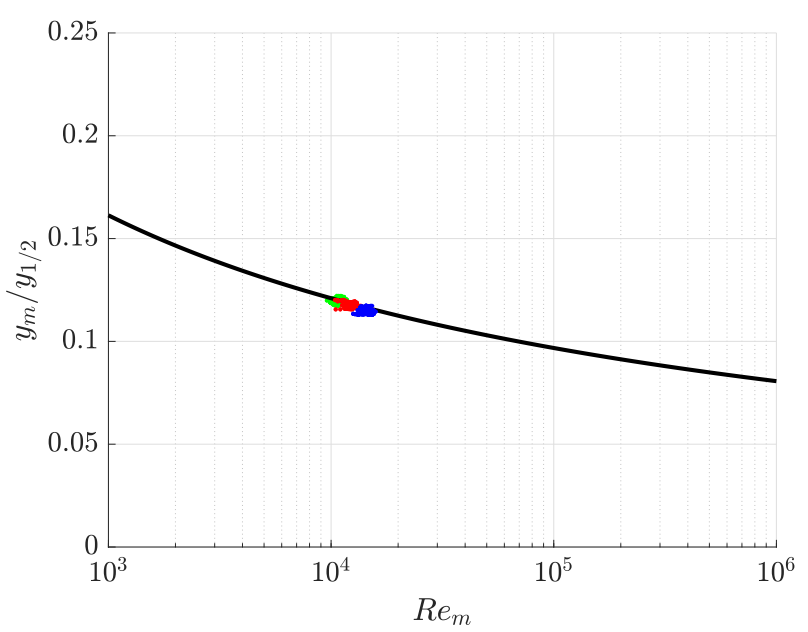

(b) Boundary layer peak velocity.

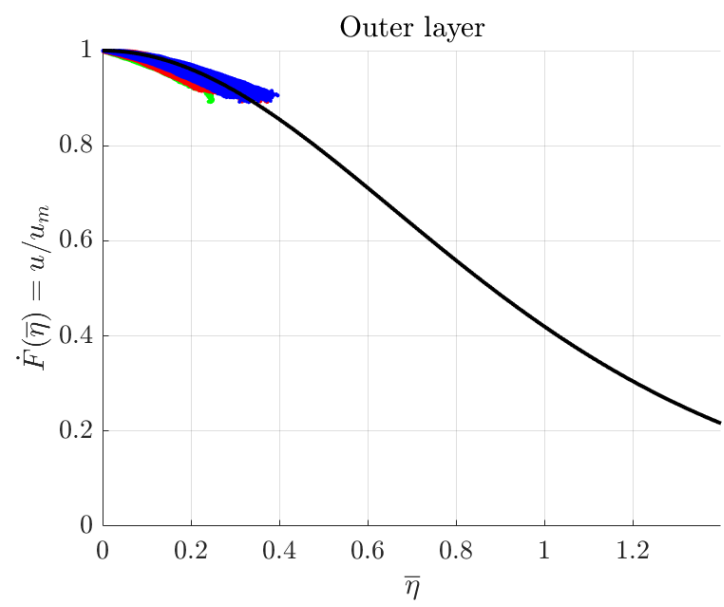

(d) Boundary layer outer layer.

Figure 8. Boundary layer properties compared to theoretical predictions of Gersten $\underline{50}$ While $y_{1 / 2}$ was not directly available from the PIV data, it was found using the facility calibration data of Kleinfelter et al $\stackrel{49}{4}$

the low-frequency reduction of surface pressure is linked with the properties of the axisymmetric boundary layer around the rods.

While experimental data suggests that canopies are effective in reducing low-frequency surface pressure fluctuations until the axisymmetric boundary layer begins to interact with the wall, empirical curve-fit of boundary layer thickness provided by Jordan $\frac{46}{1}$ may also be used in a hypothetical case (i.e. uniform free-stream without wall) to predict when the axisymmetric boundary layer around the rods would begin to interact with either the neighboring rods $(\delta=s-d / 2)$ or the wall $(\delta=h)$. The top-view of the corresponding flow field (D2S6H6) is depicted in Fig. 12 where the canopy rods are shown using black lines and the boundary layers developing over them are indicated by the blue lines. The red and blue dashed lines show the saturation points $\delta=s-d / 2$ and $\delta=h$ which were found at $x / h=11.5$ and $x / h=17.4$, respectively. The latter is closer to $x / h=15$, where a saturation in low-frequency reduction was previously found 36 Again, this suggests that the interaction between the wall and the rods boundary layer is the primary source of surface pressure reduction at low-frequencies.

Data presented thus far revealed that an axisymmetric boundary layer develops around the rods which displaces the flow above the canopies as a result of boundary layer growth. To quantify this displacement effect, we observe the location of the maximum velocity in the boundary layer $\left(y_{m}\right)$ for the baseline case (no canopy) and for the D2S6H6 case in Fig. 11(b). The growth of $y_{m}(x)$ is less steep for the baseline case than for the canopy case which is due to the flow displacement effect caused by the canopies. This effect is linked 


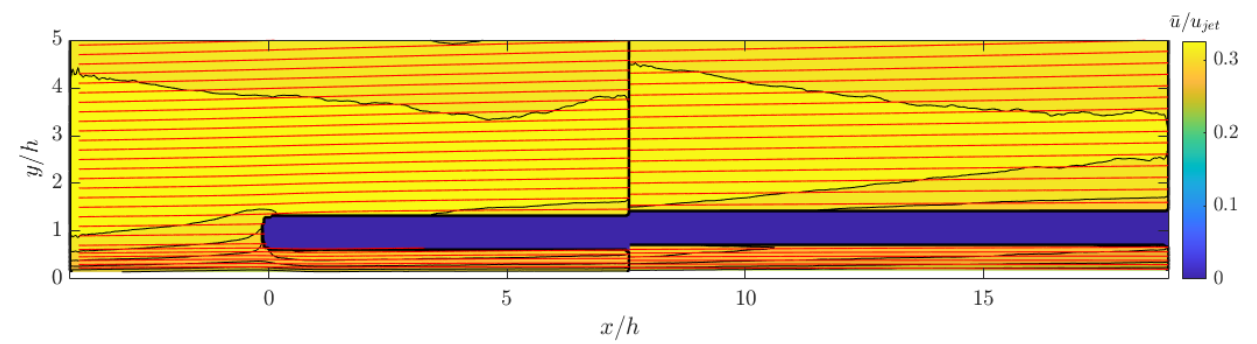

Figure 9. Streamlines and mean velocity contour plot within FOV1 and FOV2. The streamlines are indicated by the red lines. Results are shown for case D2S6H6 at $u_{j e t}=50 \mathrm{~m} / \mathrm{s}$.
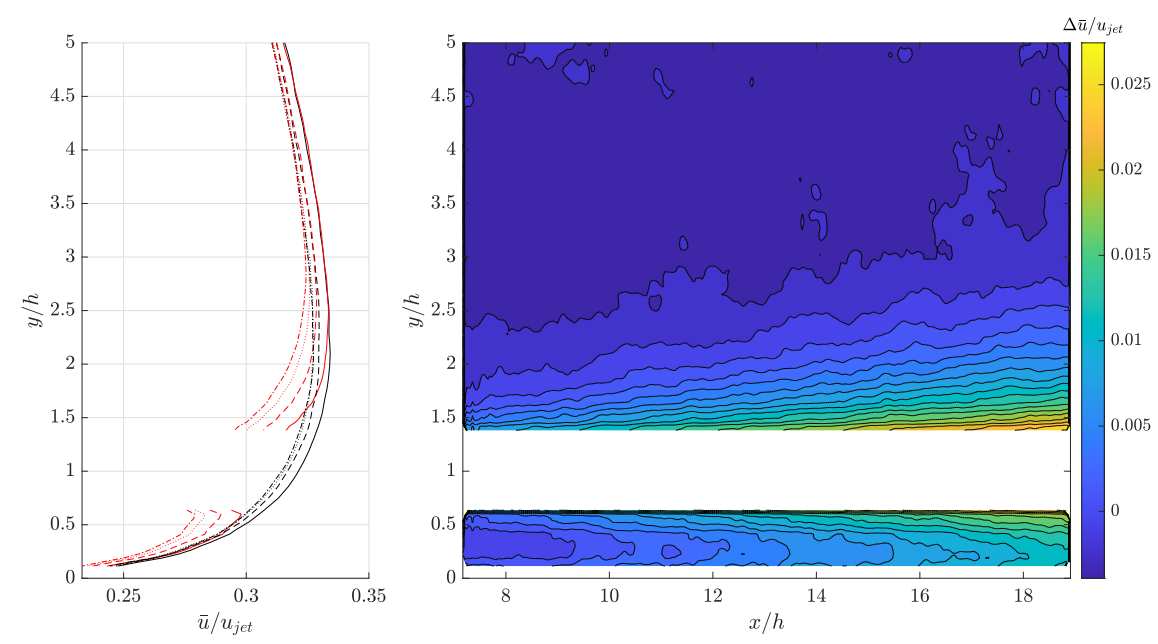

(a) Velocity profiles (left) and velocity deficit $\Delta \bar{u}=\bar{u}_{\text {baseline }}-\bar{u}_{\text {canopy }}$, (right). Legend: $-x / h=9.7,--x / h=12.7, \cdot \cdot \cdot x / h=15.7,-\cdot-x / h=18.7$, black: baseline, red: canopy.
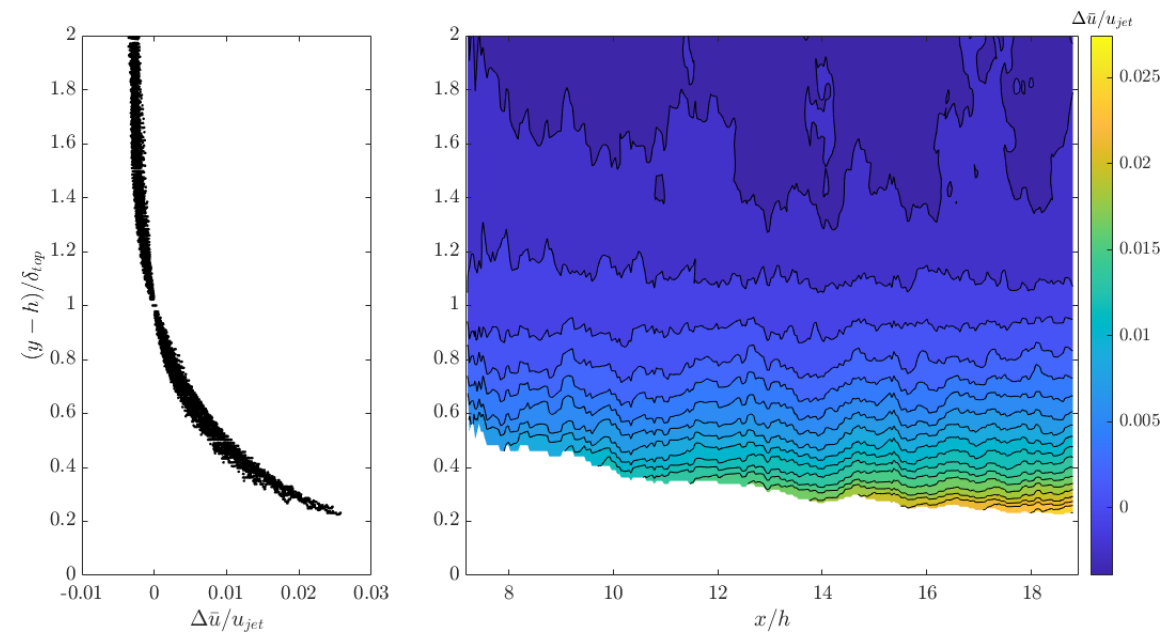

(b) Scaled velocity deficit profiles of all $x$ locations (left) and contour (right) within FOV2 above the rods.

Figure 10. Velocity deficit and its scaling around the rods for canopy D2S6H6 for FOV2 at $u_{j e t}=50 \mathrm{~m} / \mathrm{s}$.

with the blockage caused by the rods, and also with the displacement effect of the axisymmetric boundary layer and the decelerated flow below the rods. To quantify these assumptions, the velocity deficit over the area where data is available has been quantified by integrating the velocity deficit around the rods using 


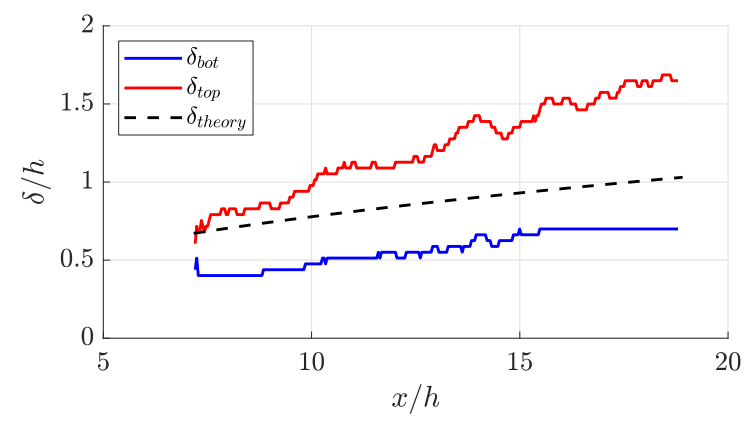

(a) Asysimmetric boundary layer thickness measured above $\left(\delta_{t o p}\right)$ and below $\left(\delta_{b o t}\right)$ the rods compared to the theoretical prediction $\delta_{\text {theory }}\left(\operatorname{Jordar}\left[\frac{46}{)}\right)\right.$.
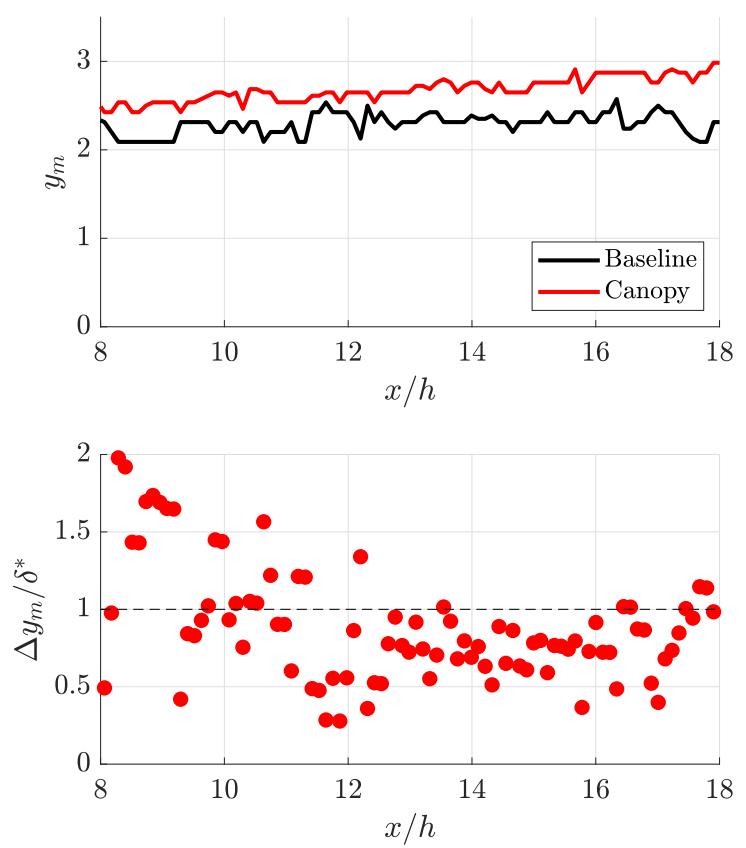

(b) Displacement of peak velocity location $y_{m}$, without (baseline) and with canopy D2S6H6 at $u_{j e t}=50 \mathrm{~m} / \mathrm{s}$.

Figure 11. Properties of the axisymmetric boundary layer developing around the rods.

$y^{\prime}=y-h$ and

$$
\delta^{*}=\int_{-\delta_{b o t}}^{\delta_{t o p}} \frac{\Delta \bar{u}}{\max (\Delta \bar{u})} \mathrm{d}\left(y^{\prime}\right) .
$$

The bottom plot in Figure 11(b) presents the canopy flow displacement effect, where $\Delta y_{m}=y_{m, c a n o p y}-$

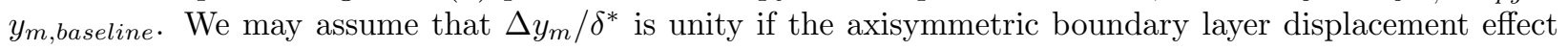
dominates the flow displacement above the rods. Indeed, the results suggest that the flow around the rods plays a major role in the blockage caused by the rods and the cross-section area of the rods does not seem to introduce a significant blockage. In fact, assuming a flat plate with equal frontal cross-section area to the rods, the equivalent thickness $(t)$ of a flat plate would be $t=d^{2} \pi /(4 s)$, i.e. $t=0.5 \mathrm{~mm}$. However, two sources of discrepancy are neglected here, namely, Eq. (18) does not include data from between the rods (as it was not available from PIV due to the optical blockage of the rods), and it does not account for the deceleration of the flow near the wall (i.e. between $y=0$ and $y^{\prime}=\delta_{b o t}$ ). Our future investigations of the canopy flow field will focus on capturing velocity data in the close vicinity of the rods (i.e. between the rods) using hot-wire anemometry and further CFD simulations to better quantify the drag and flow displacement caused by the rods.

The changes in the Reynolds stresses are now considered. Figure 13 presents the change in Reynolds stresses $\left(\Delta R_{i j}=R_{i j, c a n o p y}-R_{i j, \text { baseline }}\right)$ for cases D2S6H6 and D2S6H15 at $u_{j e t}=50 \mathrm{~m} / \mathrm{s}$. In general, introducing the canopies causes a reduction in the Reynolds stresses, which suggests the reduction of turbulent kinetic energy, hence the breakup of turbulent structures. However, two exceptions can be found from this observation. First, a slight increase is visible in $R_{u u}$ above the rods $(y / h \approx 1.75)$ for case D2S6H6 but the D2S6H15 case shows reduction only in $R_{u u}$. Second, and more importantly, the $R_{u v}$ shear stress generally increases below and decreases above the rods $(y / h=1)$. This suggests that turbulent mixing is changed when introducing the canopies to the flow. To better understand these changes, Fig. 14 presents $R_{i j}$ for the baseline and D2S6H6 cases. Results above the canopy may be seen as if a general offset has been applied to the data, because the baseline (black) and canopy (red) results are nearly parallel with each other and separated approximately by a constant offset, particularly for $R_{u v}$ and $R_{v v}$. Below the rods, on the other hand, we observe that the magnitude of $R_{u v}$ drops, which means a reduction in turbulent mixing. This is in agreement with one of our first observations regarding Fig. 9 , where the streamlines below the 


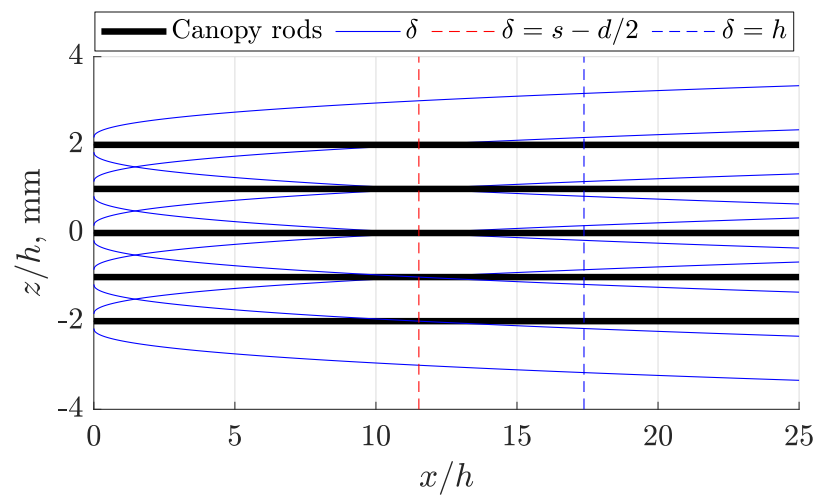

Figure 12. Top view of theoretically predicted axisymmetric turbulent boundary layer thickness developing around the rods $\left(\operatorname{Jordan}^{46}\right)$.

rods were rather parallel. This suggests that the flow below the canopies becomes less turbulent, which is also confirmed by the drop in $R_{u u}$ below the rods.

To better understand the changes below the rods, Fig. 15 presents the dimensionless velocity (Fig. 15.a)) and the friction velocity (Fig. 15(b)) for the baseline case and for case D2S6H6. From the dimensionless velocity profile $\left(u^{+}\left(y^{+}\right)\right)$, we observe that the flow below the rods still follows logarithmic scaling, which suggests that the flow in this region may follow the properties of a turbulent boundary layer flow. The friction velocity $\left(u_{\tau}\right)$ as a function of streamwise location $(x / h)$ shows that the leading edge of the rods $(x / h=0)$ has a significant effect on $u_{\tau}$ but, in agreement with our findings regarding the streamlines, this effect is localized. Past $x / h=8$ (i.e. in FOV2), the friction velocity is observed to decrease when compared to the baseline case. Still, the velocity profiles in FOV2 conform to the logarithmic layer but at a reduced friction velocity. The flow below the rods may be seen as a turbulent boundary layer with a reduced apparent friction Reynolds number $\left(R e_{\tau}=u_{\tau} y_{m} / \nu\right)$. This assumption may explain the reduction of surface pressure fluctuations at high-frequencies, which was formerly reported in Refs 36 There, the high-frequency scaling was found to be a viscous effect which suggests changes in the near-wall velocity profile. Our future investigations will focus on better understanding the changes in the flow below the rods using higher-order turbulence statistics. 

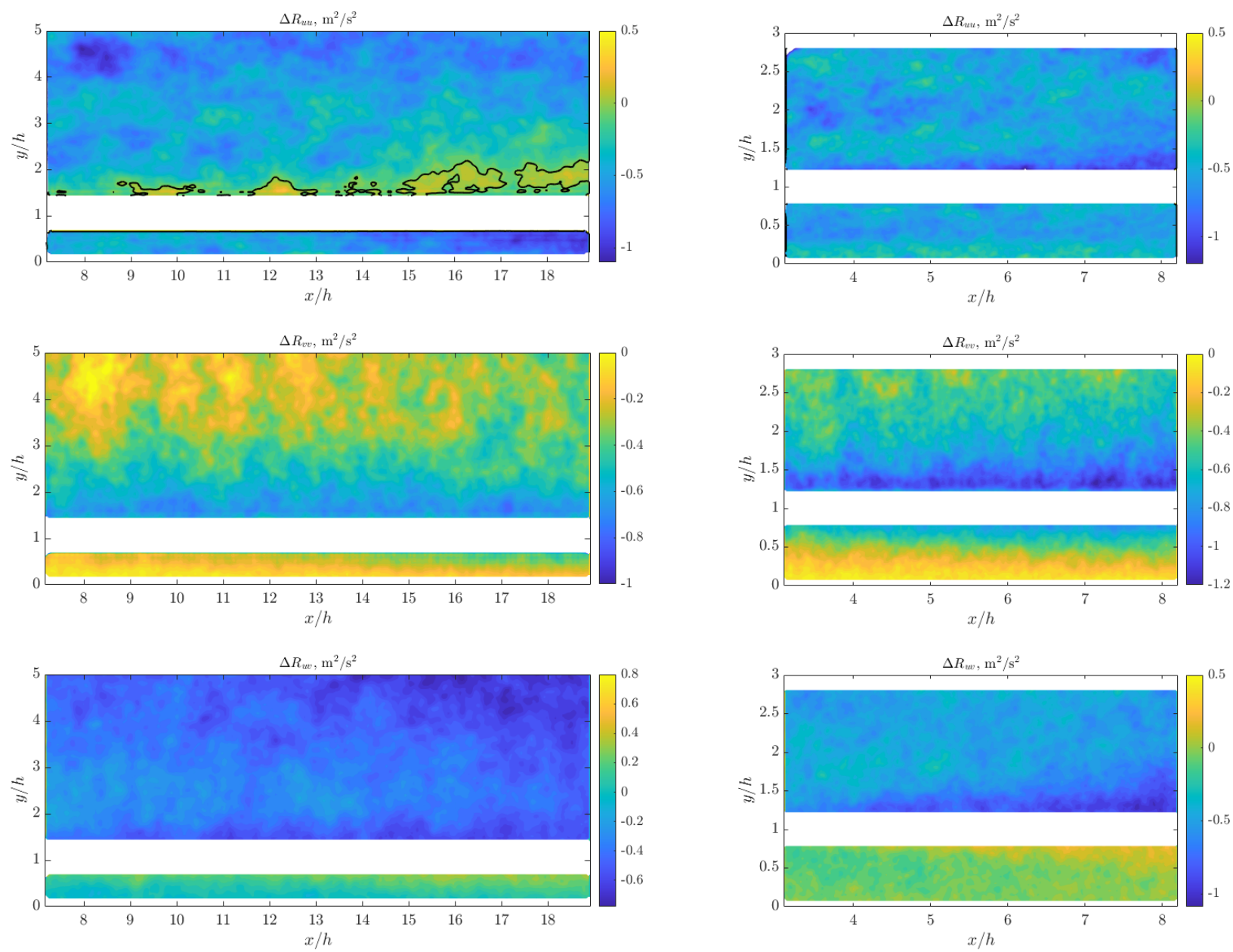

(a) Change of Reynolds shear stress contour maps $\left(\Delta R_{u u}, \Delta R_{u v}, \Delta R_{v v},\right)$ in FOV2 for D2S6H6.

(b) Change of Reynolds shear stress contour maps $\left(\Delta R_{u u}, \Delta R_{u v}, \Delta R_{v v},\right)$ in FOV2 for D2S6H15.

Figure 13. Changes in the Reynolds shear stresses when introducing canopies in the flow field. Results are shown for $u_{\text {jet }}=50 \mathrm{~m} / \mathrm{s}$.
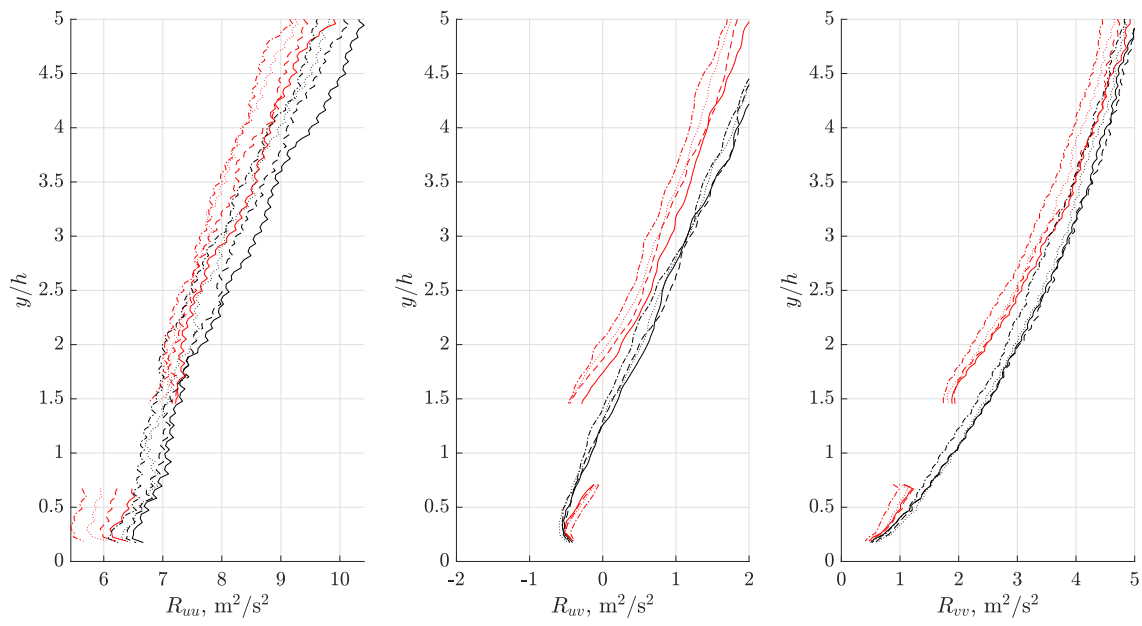

Figure 14. Reynolds shear stress around the canopy. For legend, see Fig. 10(a). 


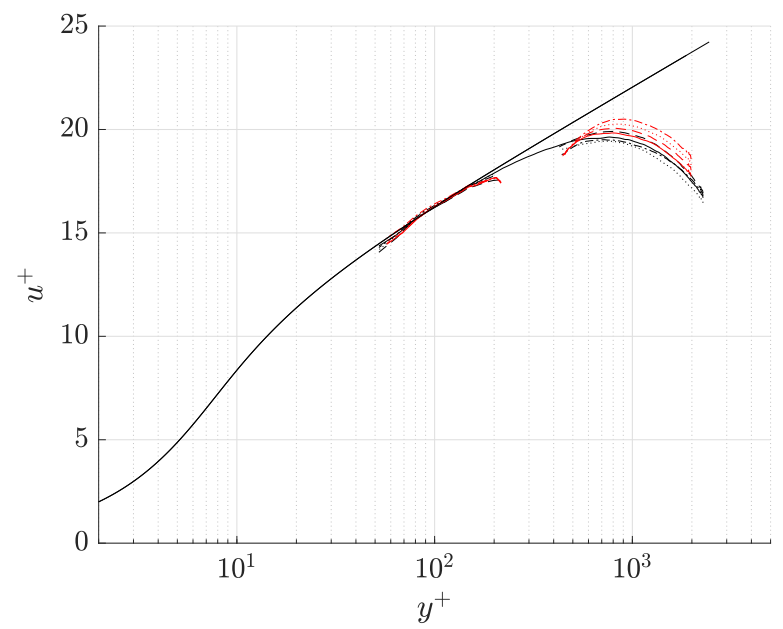

(a) Dimensionless velocity profile for the baseline case (black) and for D2S6H6 (red) at FOV2. For legend, see Fig. 10.a).

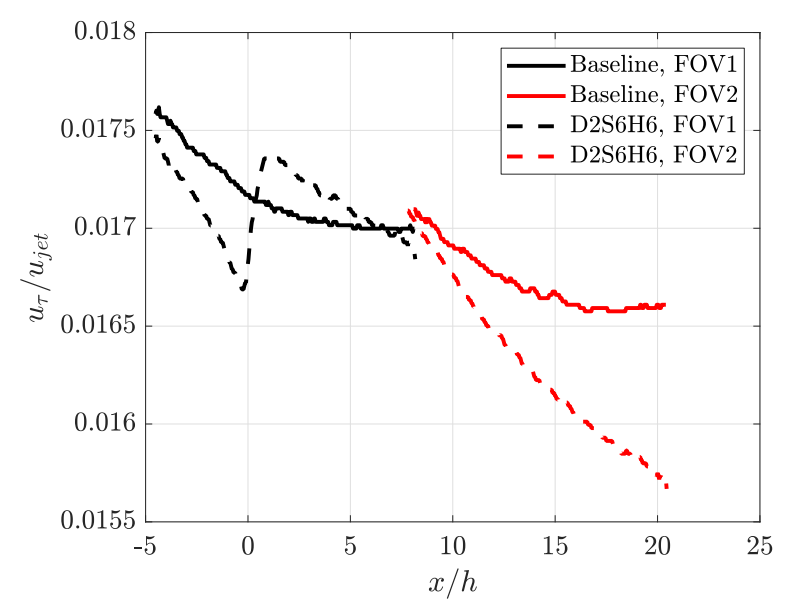

(b) Friction velocity $\left(u_{\tau}\right)$ as a function of streamwise location without and with canopies.

Figure 15. Dimensionless velocity profile and friction velocity as a function of streamwise location ( $x$ ) for case D2S6H6 at $u_{j e t}=50 \mathrm{~m} / \mathrm{s}$. 


\section{Large eddy simulation}

Results obtained from the large eddy simulation (LES) are now considered. While only a simulation using the D2S6H6 geometry is available, the data readily provides a detailed description of the flow between the rods. To explore this, we investigate mean velocity data $(\bar{u}, \bar{v}, \bar{w})$ and Reynolds stresses $\left(R_{u u}, R_{u v}, R_{v v}\right)$ at planes $z / h=0$, (mid-plane between rods), $y / h=0.5(y=3 \mathrm{~mm})$ and at $y / h=1(y=6 \mathrm{~mm})$. As a reference baseline dataset is not yet available, we consider $x / h=-3$ as a reference location (denoted using "ref." subscript), where the freestream velocity $\left(u_{\infty}\right)$ is defined as the maximum velocity.

Figures 16(a), 18(a), and 19(a) present the mean velocities at $z / h=0, y / h=0.5$, and at $y / h=1$, respectively. We observe that the rods decelerate the flow between the rods and generate an upwash flow, in agreement with the experiments. This can be seen in the $z / h=0$ plane via the $\bar{u}$ contour plots and also $\bar{u}$ at $y / h=0.5$. The rods entry condition $x / h=0$ is observed to have a localized effect in the LES data too, in agreement with the experiments. This can be seen by the increase in $\bar{u}$ and $\bar{w}$ around the leading edge of the rods within the $z / h=0$ and $y / h=0.5$ planes. The flow displacement effect caused by the rods can be observed using $\bar{v}$ contour plots in the $z / h=0$ plane, where an increasing magnitude of $\bar{v}$ can be seen past $x / h=15$. This again suggests that the axysimmetric boundary layer around the rods saturates at $x / h \approx 15$, which is in agreement with both the theoretical predictions and experimental data.

Figure 17 presents the velocity deficit $\left(\bar{u}_{d}\right)$ as contour plots and line plots at various streamwise locations. The velocity deficit is defined as $\bar{u}_{d}=\left(\bar{u}-\bar{u}_{\text {ref }}\right) / u_{\infty}$ where $\bar{u}_{\text {ref }}$ is the velocity profile at $x / h=-3$, i.e. $\bar{u}_{r e f}=\bar{u}(x / h=-3)$. Figure 17 (a) reveals that the flow is accelerated below the rods within $0<x / h<5$, which is in agreement with the increase in $u_{\tau}$ formerly observed in Fig. 15(b). The line plots shown in Figure 17(b) also reveal the same affect at $x / h=0$, and $x / h=3$. The velocity deficit line plots also reveal that there are two regions present in the velocity deficit profiles until $x / h=12$, with each region characterized with a local minimum. Namely, when observing $x / h=9$, it can be seen that there is a distinct negative velocity deficit peak at a low wall distance $(y / h<0.1)$ and another one at the rods height, $y / h \approx 1$. This again suggests that the friction velocity is reduced below the rods even before the asysimmetric boundary layer is reaching the wall.

The Reynolds stresses are now considered. Observing the Reynolds stresses in Figs. 16(b), 18(b), and 19 (b) suggest a series of changes caused by the rods in the turbulent statistics. In the $z / h=0$ plane, the upwash flow caused by the rods can be observed, and changes both below and above the rods become visible in the Reynolds stresses. Considering $x / h=-3$ as a reference, baseline condition, the rods cause a reduction of both $R_{u u}$ and $R_{v v}$ around and below $y / h=1$, which is in agreement with the experimental data. The development of the axisymmetric turbulent boundary layer may be observed at the rods height $(y / h=1)$ in Fig. 19(b), where an increase in Reynolds stresses can be seen over the surface of the rods. This again confirms the presence of an axisymmetric turbulent boundary layer over the rods.

Finally, Fig. 16(c) presents Q-criterion iso-levels colored by the streamwise velocity magnitude. The figure suggests that vortices are developing over the surface of the rods with increasing $x / h$. It may also be seen that the size of the turbulent structures around (and upstream of) the rods leading edge are larger than further downstream, again suggesting changes in the size of flow structures which may be linked with the change in turbulent mixing previously observed. 

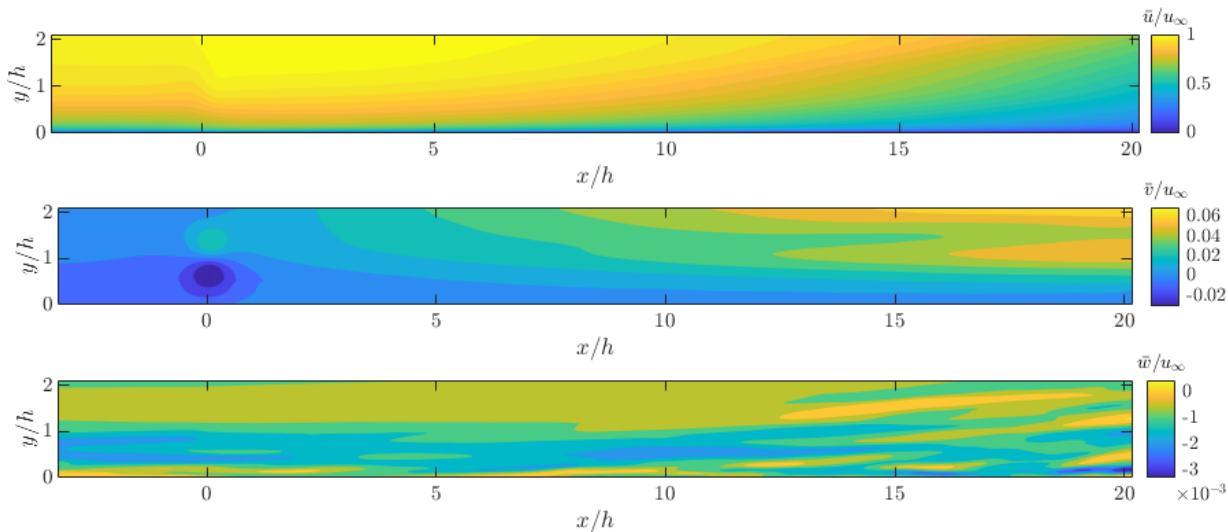

(a) Mean velocity between the rods, at $z=0$.

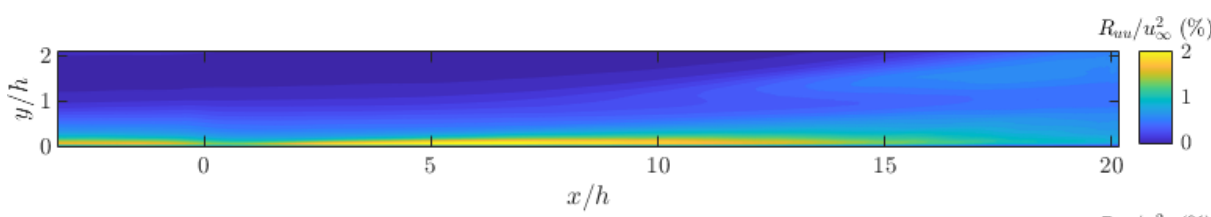

$R_{u w} / u_{\infty}^{2}(\%)$
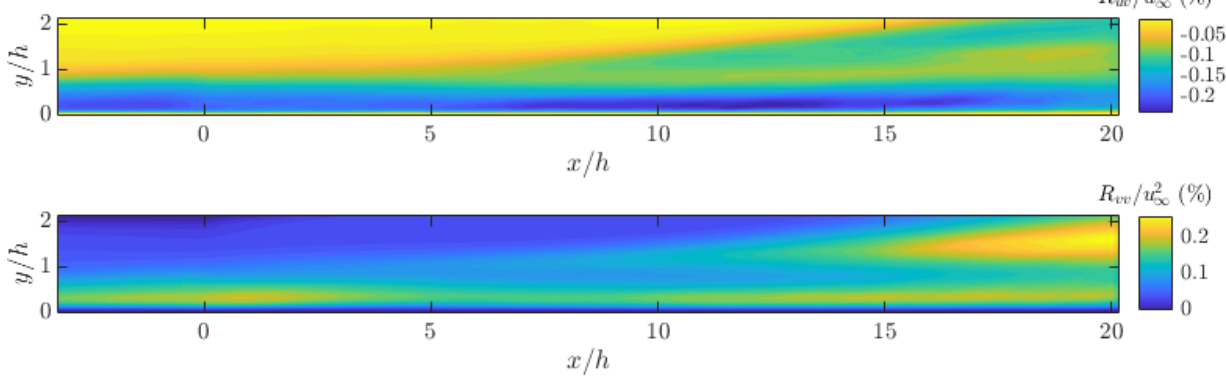

(b) Reynolds stresses between the rods, at $z=0$.

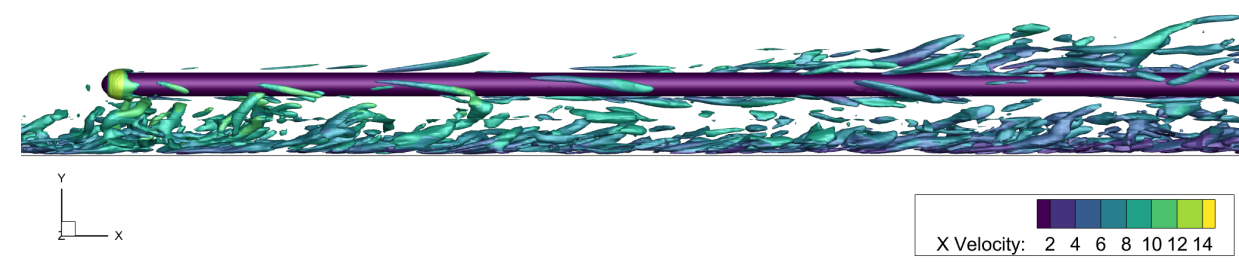

(c) Q-criterion visualized around the rods $(Q=2 e 6)$, colored by streamwise velocity $(u)$.

Figure 16. Results obtained from the large eddy simulation at the plane defined half-way between the rods $(z=0)$ and flow visualization using Q-criterion. 

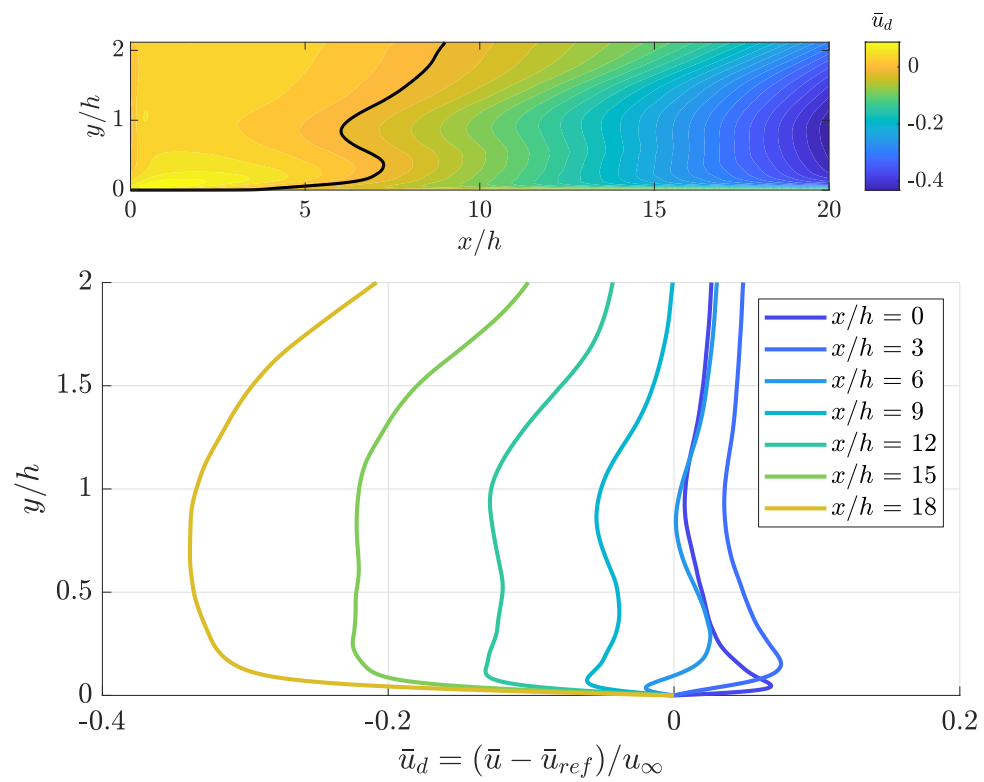

Figure 17. Velocity deficit $\left(u_{d}\right)$ as a function of streamwise location, $x / h$. The black contour line corresponds to $u_{d}=0$.
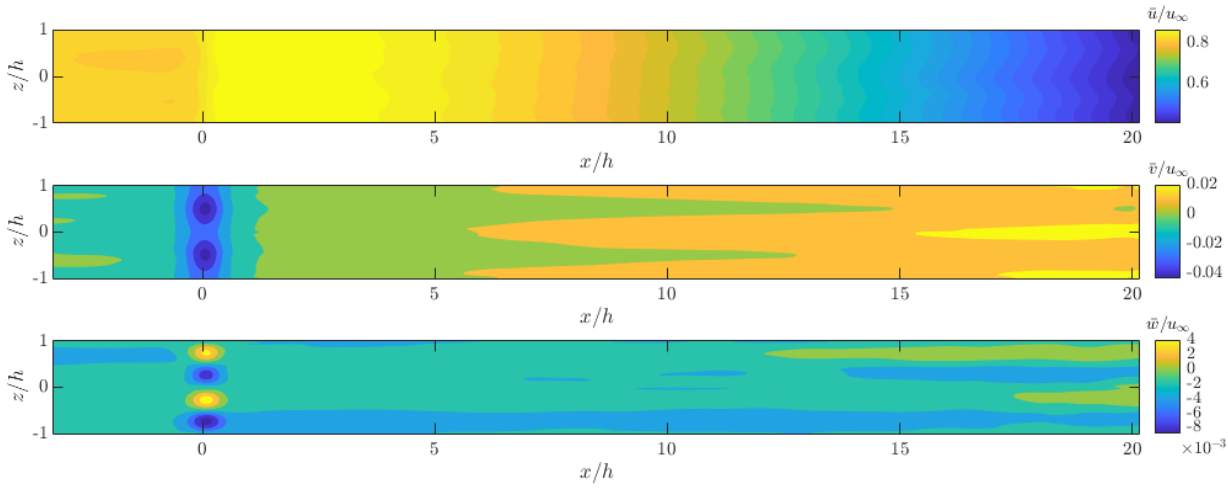

(a) Mean velocity at the mid-plane between the rods and the wall, at $y=3 \mathrm{~mm}$.
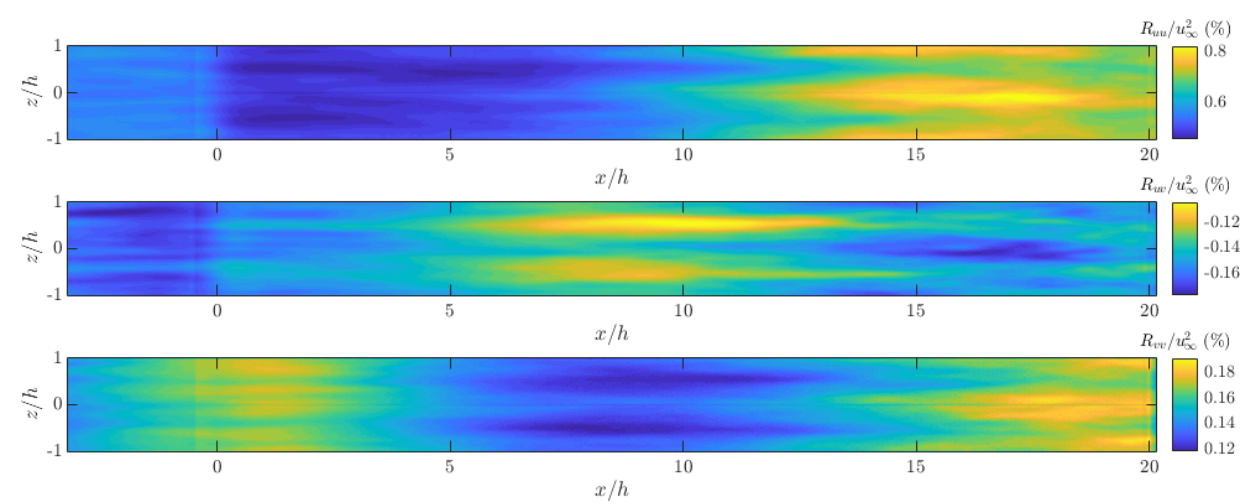

(b) Reynolds stresses at the mid-plane between the rods and the wall, at $y=3 \mathrm{~mm}$.

Figure 18. Results obtained from the large eddy simulation at the plane defined half-way between the rods and the wall $(y / h=0.5)$. 

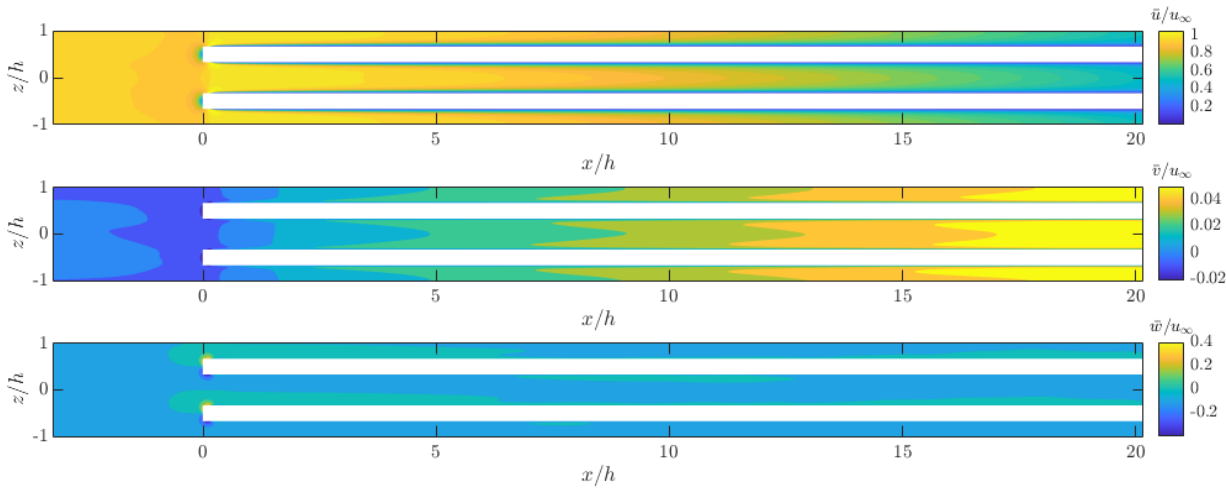

(a) Mean velocity at the rods height, at $y=6 \mathrm{~mm}$.
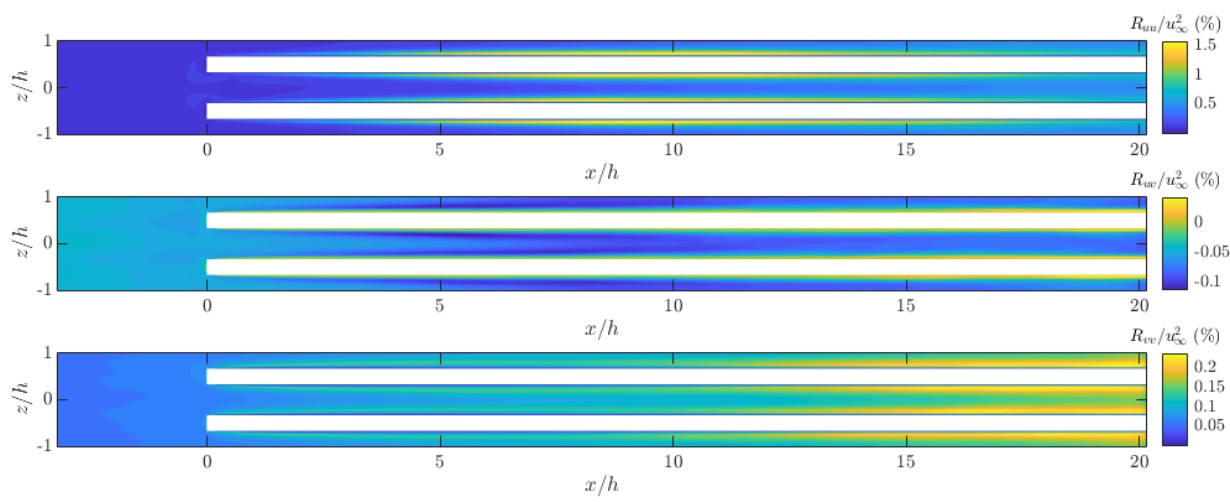

(b) Reynolds stresses at the rods height, at $y=6 \mathrm{~mm}$.

Figure 19. Results obtained from the large eddy simulation at the plane defined at the rods height $(y / h=1)$. 


\section{Conclusions and Future Work}

The flow field around a series of streamwise rods, referred to as canopies, is investigated using twodimensional time-resolved particle image velocimetry (PIV) and large eddy simulations (LES). These structures, when immersed into a turbulent boundary layer, were previously shown to efficiently reduce both low and high-frequency surface pressure fluctuations, whose underlying flow-field explanations are first investigated in the present work.

Experiments were conducted in the wall jet wind tunnel facility of Virginia Tech. PIV was used to capture the flow field for the baseline (no canopy) and two canopy configurations with rod diameter $d=2 \mathrm{~mm}$, rod spacing $s=6 \mathrm{~mm}$, and rod heights set to $h=6 \mathrm{~mm}$ and $h=15 \mathrm{~mm}$, denoted as D2S6H6 and D2S6H15, respectively. The PIV imaging was performed such that the laser sheet was located at the mid-point between two neighboring rods and positioning the line of sight of the camera with the canopy spanwise direction enabled the capture of instantaneous velocity field both below and above the rods, simultaneously.

LES simulations were also performed to resolve the flow around the rods with a geometry identical to the D2S6H6 case, but the numerical domain was restricted to the flow around a pair of rods only. A synthetic turbulence generator was used to impose a turbulent boundary layer flow well upstream of the rods, the sides of the domain were set to symmetry, the top of the domain was also set to symmetry until the flow reaches the leading edge of the rods. The boundary above the rods and the downstream face of the domain was set to outflow.

Results obtained from the baseline case (no canopy) agreed well with previous wall jet tunnel flow observations. In addition, the data was validated against the analytical model of Gersten ${ }^{[50}$ which revealed a good agreement between experiments and analytical velocity profile predictions, and wall jet boundary layer flow thickness predictions. The skin friction velocity was found to be slightly lower than theoretical predictions, which can be explained by the high Reynolds number associated with the current facility.

Scaling the velocity profiles around the rods using deficit scaling revealed that an axisymmetric turbulent boundary layer (ATBL) develops over the surface of the rods. The thickness of the ATBL was found to grow at a greater rate above the rods than below. The thickness of the ATBL below the rods was found to reach the wall at a streamwise location where a saturation was previously observed in the low-frequency surface pressure attenuation. These observations suggest that the ATBL is responsible for attenuating the pressure fluctuations at low frequencies. It was found from both PIV and LES data that the canopies act as a blockage to the flow resulting in a flow displacement effect, particularly above the rods. The displacement thickness caused by the ATBL accounted for the majority of the upward shift in the boundary layer peak velocity. This strongly suggests that the blockage caused by the ATBL dominates over the blockage caused by the rods frontal facing area.

Data from both the LES and PIV study revealed that the flow is decelerated below the rods. From the dimensionless velocity profiles and friction velocity, it was found that a logarithmic layer still exists below the rods, but with a reduced friction velocity. The streamlines depicted using the PIV data were parallel below the rods. This suggests the presence of a boundary layer-like flow with an apparent reduction of friction Reynolds number below the canopies.

Our future investigations will focus on capturing the flow field between the rods using hot-wire anemometry and capturing the flow field below the rods by orienting the FOV in a wall-parallel way. Additional LES simulations are also considered without the presence of the rods to quantify their effect on the flow field.

\section{Acknowledgments}

The authors would like to thank the National Science Foundation, in particular Dr. Ron Joslin, for their support of this research under grant CBET-1802915.

\section{References}

\footnotetext{
${ }^{1}$ Brooks, T. F., Pope, D. S., and Marcolini, M. A., "Airfoil Self-Noise and Prediction," NASA Report, Vol. 1218, 1989.

${ }^{2}$ Brooks, T. F. and Hodgson, T. H., "Trailing Edge Noise Prediction from Measured Surface Pressures," Journal of Sound and Vibration, Vol. 78(1), 1981, pp. 69-117.

${ }^{3}$ Amiet, R. K., "Noise Due to Turbulent Flow Past a Trailing Edge," Journal of Sound and Vibration, Vol. 47(3), 1976, pp. 387-393.
} 
${ }^{4}$ Amiet, R. K., "Acoustic radiation from an airfoil in a turbulent stream," Journal of Sound and Vibration, Vol. 41, No. 4, 1975, pp. 407-420.

${ }^{5}$ R. R. Parchen and Technisch Physisce Dienst, "Progress report DRAW: A prediction scheme for trailing edge noise based on detailed boundary layer characteristics," TNO Report, Vol. (HAG-RPT-980023), 1998.

${ }^{6}$ Howe, M. S., "A review of the theory of trailing edge noise," Journal of Sound and Vibration, Vol. 61, No. 3, 1978, pp. $437-465$.

${ }^{7}$ Lyu, B., Azarpeyvand, M., and Sinayoko, S., "Prediction of noise from serrated trailing edges," Journal of Fluid Mechanics, Vol. 793, 2016, pp. 556-588.

${ }^{8}$ Wang, M., "Computation of trailing-edge noise at low Mach number using LES and acoustic analogy," Centre for Turbulence Research, Annual Research Briefs, 1998, pp. 91-105.

${ }^{9}$ Wang, M. and Moin, P., "Computation of trailing-edge flow and noise using large-eddy simulation," AIAA Journal, Vol. 38, No. 12, 2000, pp. 2201-2209.

${ }^{10}$ Ewert, R. and Schroeder, W., "On the simulation of trailing edge noise with a hybrid LES/APE method," Journal of Sound and Vibration, Vol. 270, No. 3, 2004, pp. $509-524$.

${ }^{11}$ Wolf, W. R. and Lele, S. K., "Trailing-Edge Noise Predictions Using Compressible Large-Eddy Simulation and Acoustic Analogy," AIAA Journal, Vol. 50(11), 2012.

${ }^{12}$ Gruber, M., Airfoil noise reduction by edge treatments, Ph.D. thesis, University of Southampton, UK., 2012.

${ }^{13}$ Liu, X., Kamliya, H. J., Azarpeyvand, M., and Theunissen, R., "Wake Development of Airfoils with Serrated Trailing Edges," 22nd AIAA/CEAS Aeroacoustics Conference, AIAA-2016-2817.

${ }^{14}$ Chong, T., Vathylakis, A., Joseph, P., and Gruber, M., "Self-Noise Produced by an Airfoil with Nonflat Plate TrailingEdge Serrations," AIAA Journal, Vol. 51, No. 11, 2013, pp. 2665-2677.

${ }^{15}$ Chong, T. and Joseph, P., "An experimental study of airfoil instability tonal noise with trailing edge serrations," Journal of Sound and Vibration, Vol. 332, No. 24, 2013, pp. 6335-6358.

${ }^{16}$ Chong, T., Joseph, P., and Gruber, M., "Airfoil self noise reduction by non-flat plate type trailing edge serrations," Applied Acoustics, Vol. 74, No. 4, 2013, pp. 607-613.

${ }^{17}$ Gruber, M., Joseph, P., and Azarpeyvand, M., "An experimental investigation of novel trailing edge geometries on airfoil trailing edge noise reduction," 19th AIAA/CEAS Aeroacoustics Conference, AIAA-2013-2011.

${ }^{18}$ Azarpeyvand, M., Gruber, M., and Joseph, P., "An analytical investigation of trailing edge noise reduction using novel serrations," 19th AIAA/CEAS Aeroacoustics Conference, AIAA-2013-2009.

${ }^{19}$ Finez, A., Jondeau, E., Roger, M., and Jacob, M., "Broadband noise reduction with trailing edge brushes," 16th AIAA/CEAS Aeroacoustics Conference, Stockholm, Sweden, AIAA-2010-3980.

${ }^{20}$ Herr, M. and Dobrzynski, W., "Experimental Investigations in Low-Noise Trailing-Edge Design," AIAA Journal, Vol. 43(6), 2005, pp. 1167-1175.

${ }^{21}$ Ali, S. A. S., Azarpeyvand, M., and da Silva, C. R. I., "Trailing-edge flow and noise control using porous treatments," Journal of Fluid Mechanics, Vol. 850, 2018, pp. 83-119.

${ }^{22}$ Showkat Ali, S., Liu, X., and Azarpeyvand, M., "Bluff Body Flow and Noise Control Using Porous Media," 22nd AIAA/CEAS Aeroacoustics Conference, Lyon, France, AIAA-2016-2754.

${ }^{23}$ Showkat Ali, S. A., Szőke, M., and Azarpeyvand, M., "Trailing Edge Bluntness Flow and Noise Control Using Porous Treatments," 22nd AIAA/CEAS Aeroacoustics Conference, Lyon, France, AIAA-2016-2832.

${ }^{24}$ Showkat Ali, S. A., Azarpeyvand, M., Szőke, M., and Ilário da Silva, C. R., "Boundary layer flow interaction with a permeable wall," Physics of Fluids, Vol. 30, No. 8, 2018, pp. 085111.

${ }^{25}$ Liu, H., Azarpeyvand, M., Weia, J., and Qua, Z., "Tandem cylinder aerodynamic sound control using porous coating," Journal of Sound and Vibration, , No. 334, 2014, pp. 190-201.

${ }^{26}$ Rossian, L., Ewert, R., and Delfs, J. W., "Numerical investigation of porous materials for trailing edge noise reduction," International Journal of Aeroacoustics, Vol. 19, No. 6-8, 2020, pp. 347-364.

${ }^{27}$ Ai, Q., Azarpeyvand, M., Lachenal, X., and Weaver, P. M., "Aerodynamic and aeroacoustic performance of airfoils with morphing structures," Wind Energy, Vol. 19, No. 7, 2016, pp. 1325-1339.

${ }^{28}$ Szőke, M., Fiscaletti, D., and Azarpeyvand, M., "Effect of Inclined Transverse Jets on Trailing-Edge Noise Generation," Physics of Fluids, Vol. 30, No. 8, 2018, pp. 085110.

${ }^{29}$ Szőke, M., Fiscaletti, D., and Azarpeyvand, M., "Influence of boundary layer flow suction on trailing edge noise generation," Journal of Sound and Vibration, 2020, pp. 115276.

${ }^{30}$ Szőke, M., Fiscaletti, D., and Azarpeyvand, M., "Uniform flow injection into a turbulent boundary layer for trailing edge noise reduction," Physics of Fluids, Vol. 32, No. 8, 2020, pp. 085104.

${ }^{31}$ Afshari, A., Azarpeyvand, M., Dehghan, A. A., and Szőke, M., "Trailing Edge Noise Reduction Using Novel Surface Treatments," 22nd AIAA/CEAS Aeroacoustics Conference, Lyon, France, AIAA-2016-2834.

${ }^{32}$ Afshari, A., Azarpeyvand, M., Dehghan, A. A., and Szóke, M., "Three-Dimensional Surface Treatments for Trailing Edge Noise Reduction," 23rd International Congress on Sound 86 Vibration, Athens, Greece, 2016.

${ }^{33}$ Clark, I., Baker, D., Alexander, W. N., Devenport, W., Glegg, S. A., Jaworski, J., and Peake, N., "Experimental and Theoretical Analysis of Bio-Inspired Trailing Edge Noise Control Devices," 22nd AIAA/CEAS Aeroacoustics Conference, 2016, p. 3020 .

${ }^{34}$ Clark, I., Alexander, W. N., Devenport, W., Glegg, S. A., Jaworski, J., Daily, C., and Peake, N., "Bio-Inspired Trailing Edge Noise Control," 21nd AIAA/CEAS Aeroacoustics Conference, 2015, p. 2365.

${ }^{35}$ Afshari, A., Azarpeyvand, M., Dehghan, A. A., Szoke, M., and Maryami, R., "Trailing-edge flow manipulation using streamwise finlets," Journal of Fluid Mechanics, Vol. 870, 2019, pp. 617-650.

${ }^{36}$ Gonzalez, A., Glegg, S. A., Hari, N., and Devenport, W. J., "Fundamental studies of the mechanisms of pressure shielding," 25th AIAA/CEAS Aeroacoustics Conference, 2019, p. 2403. 
${ }^{37}$ Hari, N. N., Szoke, M., Devenport, W., and Glegg, S., "Understanding Pressure Shielding by Canopies," 2021 AIAA SciTech Forum, , No. AIAA 2021-0817, AIAA-2021-0817.

${ }^{38}$ Jimenez, I., Glegg, S. A., and Devenport, W. J., "The effect of shear sheltering on trailing edge noise," AIAA Aviation 2020 Forum, 2020, p. 2515.

${ }^{39}$ Hunt, J. and Durbin, P., "Perturbed vortical layers and shear sheltering," Fluid dynamics research, Vol. 24, No. 6, 1999, pp. 375 .

${ }^{40}$ Jacobs, R. G. and Durbin, P. A., "Shear sheltering and the continuous spectrum of the Orr-Sommerfeld equation," Physics of Fluids, Vol. 10, No. 8, 1998, pp. 2006-2011.

${ }^{41}$ Zaki, T. A. and Saha, S., "On shear sheltering and the structure of vortical modes in single-and two-fluid boundary layers," 2009.

${ }^{42}$ Hernon, D., Walsh, E. J., and McELIGOT, D. M., "Experimental investigation into the routes to bypass transition and the shear-sheltering phenomenon," Journal of Fluid Mechanics, Vol. 591, 2007, pp. 461.

${ }^{43}$ Lueptow, R. M., Leehey, P., and Stellinger, T., "The thick, turbulent boundary layer on a cylinder: Mean and fluctuating velocities," The Physics of fluids, Vol. 28, No. 12, 1985, pp. 3495-3505.

${ }^{44}$ Tutty, O. R., "Flow along a long thin cylinder," Journal of Fluid Mechanics, Vol. 602, 2008, pp. 1-37.

${ }^{45}$ Piquet, J. and Patel, V., "Transverse curvature effects in turbulent boundary layer," Progress in aerospace sciences, Vol. 35, No. 7, 1999, pp. 661-672.

${ }^{46}$ Jordan, S. A., "On the axisymmetric turbulent boundary layer growth along long thin circular cylinders," Journal of Fluids Engineering, Vol. 136, No. 5, 2014.

${ }^{47}$ Cipolla, K. and Keith, W., "High Reynolds number thick axisymmetric turbulent boundary layer measurements," Experiments in Fluids, Vol. 35, No. 5, 2003, pp. 477-485.

${ }^{48}$ Kumar, P. and Mahesh, K., "Large-eddy simulation of flow over an axisymmetric body of revolution," Journal of Fluid Mechanics, Vol. 853, 2018, pp. 537-563.

${ }^{49}$ Kleinfelter, A. W., Repasky, R., Hari, N., Letica, S., Vishwanathan, V., Organski, L., Schwaner, J., Alexander, W. N., and Devenport, W. J., "Development and Calibration of a new Anechoic Wall Jet Wind Tunnel," AIAA Scitech 2019 Forum, 2019, p. 1936.

${ }^{50}$ Gersten, K., "The asymptotic downstream flow of plane turbulent wall jets without external stream," Journal of Fluid Mechanics, Vol. 779, 2015, pp. 351.

${ }^{51}$ George, W. K., Abrahamsson, H., Eriksson, J., Karlsson, R. I., Löfdahl, L., and Wosnik, M., "A similarity theory for the turbulent plane wall jet without external stream," Journal of Fluid Mechanics, Vol. 425, 2000, pp. 367-411.

${ }^{52}$ Gnanamanickam, E. P., Bhatt, S., Artham, S., and Zhang, Z., "Large-scale motions in a plane wall jet," Journal of Fluid Mechanics, Vol. 877, 2019, pp. 239-281.

${ }^{53}$ Choi, H. and Moin, P., "Grid-point requirements for large eddy simulation: Chapman's estimates revisited," Physics of Fluids, Vol. 24, No. 1, 2012, pp. 1-6.

${ }^{54}$ Smagorinsky, J., "General Circulation Experiments With the Primitive Equations," Monthly Weather Review, Vol. 91, No. 3, 1963.

${ }^{55}$ Nicoud, F. and Ducros, F., "Subgrid-scale stress modelling based on the square of the velocity gradient tensor," Flow, Turbulence and Combustion, Vol. 62, 1999, pp. 183-200.

${ }^{56}$ Smirnov, A., Shi, S., and Celik, I., "Random flow generation technique for large eddy simulations and particle-dynamics modeling," Journal of Fluids Engineering, Transactions of the ASME, Vol. 123, No. 2, 2001, pp. 359-371. 